\title{
Assessment of mismatch repair deficiency, CDX2, beta-catenin and E-cadherin expression in colon cancer: molecular characteristics and impact on prognosis and survival - an immunohistochemical study
}

\author{
Carmen Stanca Melincovici ${ }^{1)}$, Adina BianCa BoşCA ${ }^{1)}$, Sergiu ŞuŞMan ${ }^{1,2)}$, AnCUta Cutaş ${ }^{3)}$,

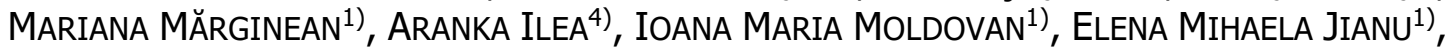 \\ Maria Adriana NeaG ${ }^{5)}$, Adriana-Elena BulboaCă6) ${ }^{6}$, Carmen Mihaela Mihu ${ }^{1)}$ \\ 1) Discipline of Histology, Department of Morphological Sciences, Iuliu Haţieganu University of Medicine and Pharmacy, \\ Cluj-Napoca, Romania \\ 2) Department of Pathology, IMOGEN Research Center, Cluj-Napoca, Romania \\ 3) Discipline of Medical Informatics and Biostatistics, Iuliu Hatieganu University of Medicine and Pharmacy, Cluj-Napoca, \\ Romania \\ 4) Department of Oral Rehabilitation, Iuliu Hatieganu University of Medicine and Pharmacy, Cluj-Napoca, Romania \\ ${ }^{5)}$ Discipline of Pharmacology, Toxicology and Clinical Pharmacology, Department of Functional Biosciences, \\ Iuliu Hatieganu University of Medicine and Pharmacy, Cluj-Napoca, Romania \\ ${ }^{6)}$ Department of Functional Biosciences, Iuliu Hatieganu University of Medicine and Pharmacy, Cluj-Napoca, Romania
}

\begin{abstract}
Microsatellite instability (MSI) or the deficiency of mismatch repair (MMR) proteins is one of the molecular pathways of colorectal tumorigenesis and may have important clinical implications in predicting the treatment response. We evaluated the relationship between clinicopathological features and MMR proteins [mutL homologue 1 (MLH1), mutS homologue 2 (MSH2), mutS homologue 6 (MSH6), postmeiotic segregation increased 2 (PMS2)], adhesion molecules (E-cadherin, beta-catenin) and caudal-type homeobox 2 (CDX2) in 31 patients with colon adenocarcinoma, using immunohistochemistry. We also aimed to assess the prognostic value of the studied proteins. MLH1 loss was correlated to PMS2 loss $(p=0.006)$ and MSH2 loss $(p=0.023)$; MSH2 loss was significantly associated to MSH6 loss $(p=0.011)$. Tumors with MSH6 loss, together with tumors with PMS2 loss, covered all the patients with MSI status. We found a significant correlation between MSI tumors and mucinous histological type $(p=0.03)$, but no significant associations with other clinicopathological features or with survival rate. There was a significant correlation between E-cadherin expression and differentiation degree $(p=0.018)$ and between beta-catenin expression and lymph node invasion $(p=0.046)$. No significant association between CDX2 loss and any clinical or pathological features was found $(p>0.05)$. No significant differences were identified in overall survival according to E-cadherin, beta-catenin or CDX2 expression $(p>0.05)$. In our study, PMS2 loss was significantly correlated with CDX2 loss $(p=0.03)$. In conclusion, the molecular analysis of biological markers for colon cancer may be important for patient stratification, in order to select the optimal treatment algorithm. Our results suggest that probably the double panel (MSH6 and PMS2) is enough to detect the MSI status, instead of using the quadruple panel.
\end{abstract}

Keywords: colon cancer, microsatellite instability, mismatch repair proteins, CDX2, adhesion molecules.

\section{Introduction}

Despite the numerous current diagnostic and therapeutic approaches, colorectal cancer (CRC) is a major public health problem, globally being the third most prevalent cancer in both men and women [1] and the second cause of mortality [1-4].

In Romania, according to Global Cancer Observatory, colon cancer was the second in terms of both incidence (13.3\%), and mortality (17\%), after lung cancer [5].

However, the incidence $(0.6 \%$ per year $)$ and the number of deaths ( $18 \%$ per year in men and $1.6 \%$ per year in women) has declined in the recent years $[1,3]$, which proves the efficiency of the screening and therapeutic methods and a better understanding of the molecular mechanisms involved in colon carcinogenesis $[2,3]$.
It is unanimously accepted that $\mathrm{CRC}$ is a heterogeneous disease, with various clinical and pathological characteristics, different prognosis, and response to treatment, even in patients with the same tumor, node, metastasis (TNM) stage [6]. This may be due to the various molecular mechanisms implicated in colon tumorigenesis [4].

Two pathways are recognized in colon carcinogenesis: the chromosomal instability (CIN) and microsatellite instability (MSI) [3, 7-10].

MSI occurs when the mismatch repair $(M M R)$ genes are inactivated; under normal conditions, these genes repair the spontaneous defects that occur during deoxyribonucleic acid (DNA) replication [11]. Because of MMR genes inactivation, the cell becomes more vulnerable to further mutations. These mutations affect the short DNA sequences,

This is an open-access article distributed under the terms of a Creative Commons Attribution-NonCommercial-ShareAlike 4.0 International Public License, which permits unrestricted use, adaptation, distribution and reproduction in any medium, non-commercially, provided the new creations are licensed under identical terms as the original work and the original work is properly cited. 
called microsatellite regions [12]. Microsatellites are tandem repetitive sequences of DNA nucleotides [e.g., $(\mathrm{CA}) \mathrm{n}]$, normally found in the human genome that are susceptible to insertions/deletions mutations during DNA replication [12]. The lack of efficient $M M R$ genes enables permanent changes in the length of these repetitive sequences or microsatellites (insertion or deletion of nucleotides), a process called MSI [3, 4, 11-13].

Tumors with MSI are found not only in the colon, but can also occur, at a lower rate, as extracolonic malignancies, particularly endometrial cancer $[3,6,11$, 14-17], breast cancer [11], ovarian cancer [6, 16-18], pancreatic cancer [11], stomach cancer [6, 11, 19], prostate and urinary bladder cancer $[6,11,20,21]$, head and neck squamous cell carcinoma [6], non-small cell lung cancer [20, 22], melanoma [23], etc.

$M M R$ genes involved in the development of colorectal MSI tumors are mutL homologue 1 (MLH1) gene, mutS homologue 2 (MSH2) gene, mutS homologue 6 (MSH6) gene or postmeiotic segregation increased 2 (PMS2) gene $[11,24]$. Inactivation of $M L H 1$ and $M S H 2$ accounts for over $90 \%$ of deficient MMR (dMMR) cases [25].

The $M M R$ genes inactivation can be inherited, in hereditary nonpolyposis colorectal cancer (HNPCC) or Lynch syndrome, when germline mutations block the expression of $M M R$ genes, usually $M L H 1$ or $M S H 2(85 \%$ of cases). In $12-15 \%$ of cases, the genes inactivation can be acquired by epigenetic changes, e.g., in sporadic cancer, when DNA repair genes are inactivated by methylation $[3,4,6]$. MLH1 hypermethylation is an epigenetic change that could be the main cause of $M M R$ genes inactivation in sporadic cancer (70-95\% of cases) $[4,6,11,12,26]$; it can also be associated, in approximately $50 \%$ of cases, with mutations of oncogenic BRAF V600E gene [3, 6, 24]. In contrast, in hereditary cancer, there are no $B R A F$ mutations, which could be an exclusion criterion for Lynch syndrome [3, 4, 25].

In order to increase the MMR system stability, the $M M R$ genes work in pairs, forming heterodimers $M L H 1 /$ PMS2 and MSH2/MSH6 [12, 24]. MLH1 is responsible for the stability of $P M S 2$, so, in cases with combined loss of $M L H 1$ and $P M S 2$, the defective gene is $M L H 1$ $[25,26]$. A similar process appears with $M S H 2 / M S H 6$, in this situation $M S H 2$ stabilizing the complex [24, 26]. Isolated loss of MSH6 reveals defective MSH6 gene, whereas loss of both protein expression (MSH2 and MSH6) usually indicates that the defect is within $\mathrm{MSH} 2$ [12, 24-26].

The MSI tumor status can be revealed either by molecular genetic techniques, such as polymerase chain reaction (PCR), or by immunohistochemistry, in order to assess the MMR protein expression [3, 27]. The two techniques have been proven to have a high correlation degree $[6,25]$; immunohistochemistry is frequently used, since it is more accessible and cost-effective [26].

Tumors that lack MMR proteins are defined as dMMR or MSI tumors, while tumors that express MMR proteins, are regarded as microsatellite stable (MSS) tumors [25, 26].

Many other biological markers, such as caudal-type homeobox 2 (CDX2) or adhesion molecules (E-cadherin, beta-catenin), have been blamed to be involved in colon cancer progression and invasion.
E-cadherin is a transmembrane cell adhesion molecule, important in epithelial cells development and growth. It is responsible for epithelial cell cohesion and is functionally necessary for epithelial tissue integrity [28-30]. Pathological loss of E-cadherin function is associated with epithelialto-mesenchymal transition (EMT), tumor progression and metastasis [30-32]. Beta-catenin is part of the adherens junctions that binds to cytoplasmatic domain of E-cadherin, thus forming cell-to-cell adhesion. Beta-catenin promotes cell differentiation, proliferation, migration, and invasion $[33,34]$.

CDX2 is a transcription factor involved in differentiation and proliferation of intestinal epithelial cells, mainly in the distal part of the intestine. Loss of CDX2 expression was reported to be involved in progression of some sporadic CRCs [35-39].

\section{Aim}

In the present study, we aimed to analyze the relationship between patient clinicopathological features and immunoexpression of several biological markers, supposed to be involved in colon cancer progression and invasion, such as: MMR proteins (MLH1, MSH2, MSH6, PMS2), adhesion molecules (E-cadherin, beta-catenin), and CDX2. We also aimed to evaluate the correlation between all the analyzed proteins and survival rate.

\section{a Patients, Materials and Methods}

A total of 31 patients who underwent surgical resection for colon cancer at the $3^{\text {rd }}$ Surgical Clinic, Prof. Dr. Octavian Fodor Regional Institute of Gastroenterology and Hepatology, Cluj-Napoca, Romania, between 2005 and 2006, were included in this study. This is a retrospective study therefore the status of cancer specimens (sporadic or inherited) cannot be specified. No neoadjuvant chemotherapy was applied. The Ethics Committee of Prof. Dr. Octavian Fodor Regional Institute of Gastroenterology and Hepatology, Cluj-Napoca, had approved this study.

Both histopathological and immunohistochemical (IHC) studies were performed in the Department of Pathology, Prof. Dr. Ion Chiricuţă Institute of Oncology, Cluj-Napoca.

Firstly, the $10 \%$ formalin-fixed tissue samples were embedded in tissue microarray (TMA) paraffin blocks, cut in $5 \mu \mathrm{m}$ thick sections, and stained with HematoxylinEosin (HE) for routine examination. The microscopic analysis was performed by two anatomopathologists and the histological type was established according to the 2010 World Health Organization (WHO) Classification of Tumours of the Digestive System [40, 41]. We evaluated the tumor location, histological type, tumor differentiation degree, lymph node metastasis, perineural and vascular invasion, tumor necrosis, local invasion, peritumoral inflammatory infiltrate, etc. Tumor stage was established according to American Joint Committee on Cancer (AJCC, $8^{\text {th }}$ edition) classification of colon cancer [42]. Mucinous adenocarcinoma was diagnosed if the tumor had more than $25 \%$ mucus pools.

Subsequently, the IHC determinations were carried out, using the Streptavidin-Biotin complex. IHC used primary monoclonal antibodies against: MLH1 (Novocastra, clone ES05, 1/100 dilution), PMS2 (Novocastra, clone M0R4G, 
1/100 dilution), MSH2 (Novocastra, clone 25D12, 1/100 dilution), MSH6 (Novocastra, clone PU29, 1/100 dilution), beta-catenin (Novocastra, clone 17C2, 1/80 dilution), E-cadherin (Novocastra, clone 36B5, 1/80 dilution), and CDX2 (Novocastra, clone EP25, 1/50 dilution).

\section{Assessment of protein expression}

The sections were examined with an Olympus CKX 41 microscope.

Loss of MMR protein expression was considered when nuclear staining was completely absent in the tumor cells. The positive internal control was considered the nuclear staining in normal colonic mucosa, stromal cells, and lymphocytic infiltration. The normal expression of all four MMR proteins (MLH1, MSH2, MSH6, and PMS2) in tumor cells was considered to be MSS.

For beta-catenin, the positive control was the membranous staining in normal colonic mucosa, whereas in the tumor cells there was an aberrant nuclear/ cytoplasmatic expression. E-cadherin was identified as a normal membranous expression in normal colonic mucosa (positive control). In tumor cells, E-cadherin stained as a diffuse cytoplasmic and membranous signal. To evaluate the beta-catenin and E-cadherin immunostaining, we assessed the percentage of positive cells out of 100 visible cells in the microscopic field, while the intensity score represented the average staining intensity $(0-$ negative, 1 - weak, 2 - intermediate, and 3 - strong) [28].

Nuclear CDX2 expression was seen in normal and neoplastic colonic mucosa. All the colonic tumors that had a nuclear expression of CDX2 were scored CDX2 positive, and the tumors that lacked nuclear CDX2 expression were scored CDX2 negative.

The median survival time for the studied group was $32.26 \pm 8.73$ months. Survival data was obtained using the National Administrative Databases (Computerized Population Register Service).

\section{Statistical analysis}

For statistical analysis, version 25 for Widows of the Statistical Package for the Social Sciences (SPSS) statistical processing program was used. The normality of data distribution was assessed with Kolmogorov-Smirnov test. The association between qualitative variables was observed using $\chi^{2}$ (chi-squared) test, Fisher's exact test, and relative risk (RR). Non-parametric tests for correlations (Spearman,
Mann-Whitney $U$-test for independent samples) were used for the variables which did not follow a normal distribution. For the description of the data contingency tables, frequency, average and standard deviation were used. The $p$-value was considered significant when $\leq 0.05$.

\section{ㅁ Results}

The total number of 31 patients included $15(48.39 \%)$ men and 16 (51.61\%) women; the age ranged from 19 to 76 years, with a mean age of $63 \pm 11.71$ years. Five (16.12\%) patients were less than 50 years. Among the 31 samples of colon adenocarcinomas, $48.39 \%$ were located in the right colon, $67.74 \%$ were intestinal type, and $32.26 \%$ were considered mucinous type (with more than $25 \%$ mucus pools). Most of the tumors (48.39\%) were moderately differentiated, $29.03 \%$ were well differentiated, and $22.58 \%$ of tumors were poorly differentiated.

Regarding the tumor stage, according to the TNM staging of CRC [42], most of the patients (54.8\%) were TNM stage III and $9.67 \%$ of patients had distant metastases. Lymph node metastases were detected in $58.06 \%$ of cases; both venous and perineural invasion were observed in $22.58 \%$ of cases. Tumor necrosis and peritumoral lymphocytic infiltrate were present in $38.70 \%$, respectively $45.16 \%$ of cases. Local invasion in nearby organs was detected in only $6.45 \%$ of cases. The follow-up period was 40 months; the median survival time was $32.26 \pm 8.73$ months.

IHC analysis of MMR proteins detected a loss of expression for one or more MMR proteins in $26.81 \%$ (8/31 patients). Among all patients with dMMR tumors, individual or combined loss for MLH1, MSH2, MSH6 and PMS2 proteins was noticed in 75\% (6/8 patients), $50 \%(4 / 8), 50 \%(4 / 8)$, and $62.5 \%(5 / 8)$, respectively (Figures 1-4).

Combined loss of MLH1 and PMS2 (MLH1/PMS2 loss status) was detected in $62.5 \%$ (5/8 patients) of dMMR tumors and combined loss of MSH2 and MSH6 (MSH2/MSH6 loss status) in 75\% (6/8 patients) of MSI tumors. Loss of MSH6, without MSH2 loss was seen in $25 \%$ (2/8 patients) MSI patients, but we did not find any tumors with isolated loss of PMS2, without MLH1 loss. Tumors with MSH6 loss, together with tumors with PMS2 loss, covered all the dMMR tumors.

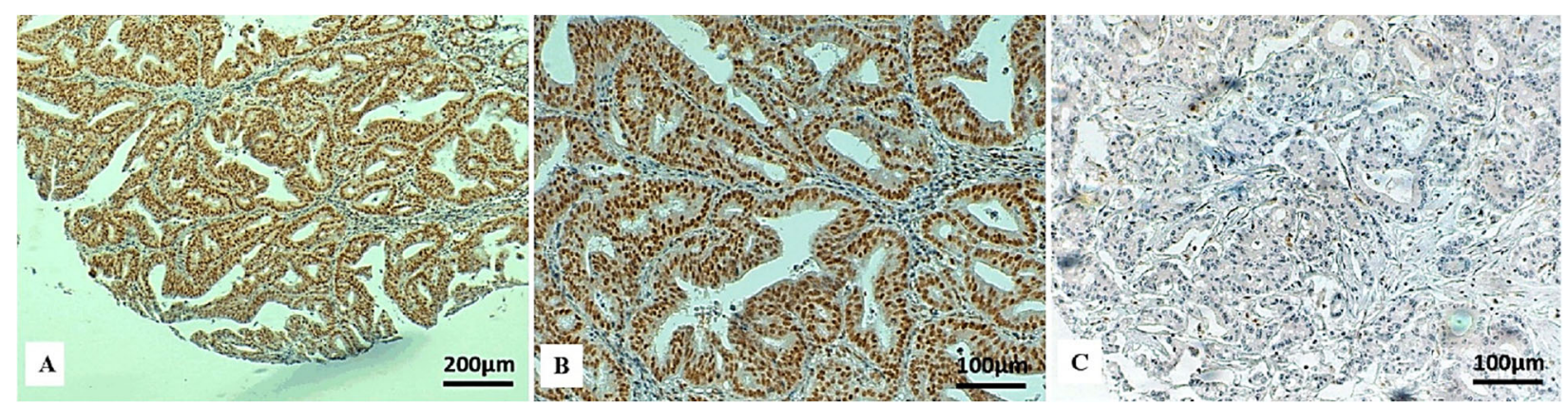

Figure 1 - Immunohistochemical staining for MLH1 in colon cancer: (A) Positive nuclear immunostaining for MLH1 in tumor cells and normal intestinal mucosa (upper right corner) $(\times 100)$; (B) Strong nuclear immunoreactivity for MLH1 in tumor cells (×200); (C) MLH1 loss in tumor cells, with positive internal control of stromal lymphocytes (×200). MLH1: mutL homologue 1. 


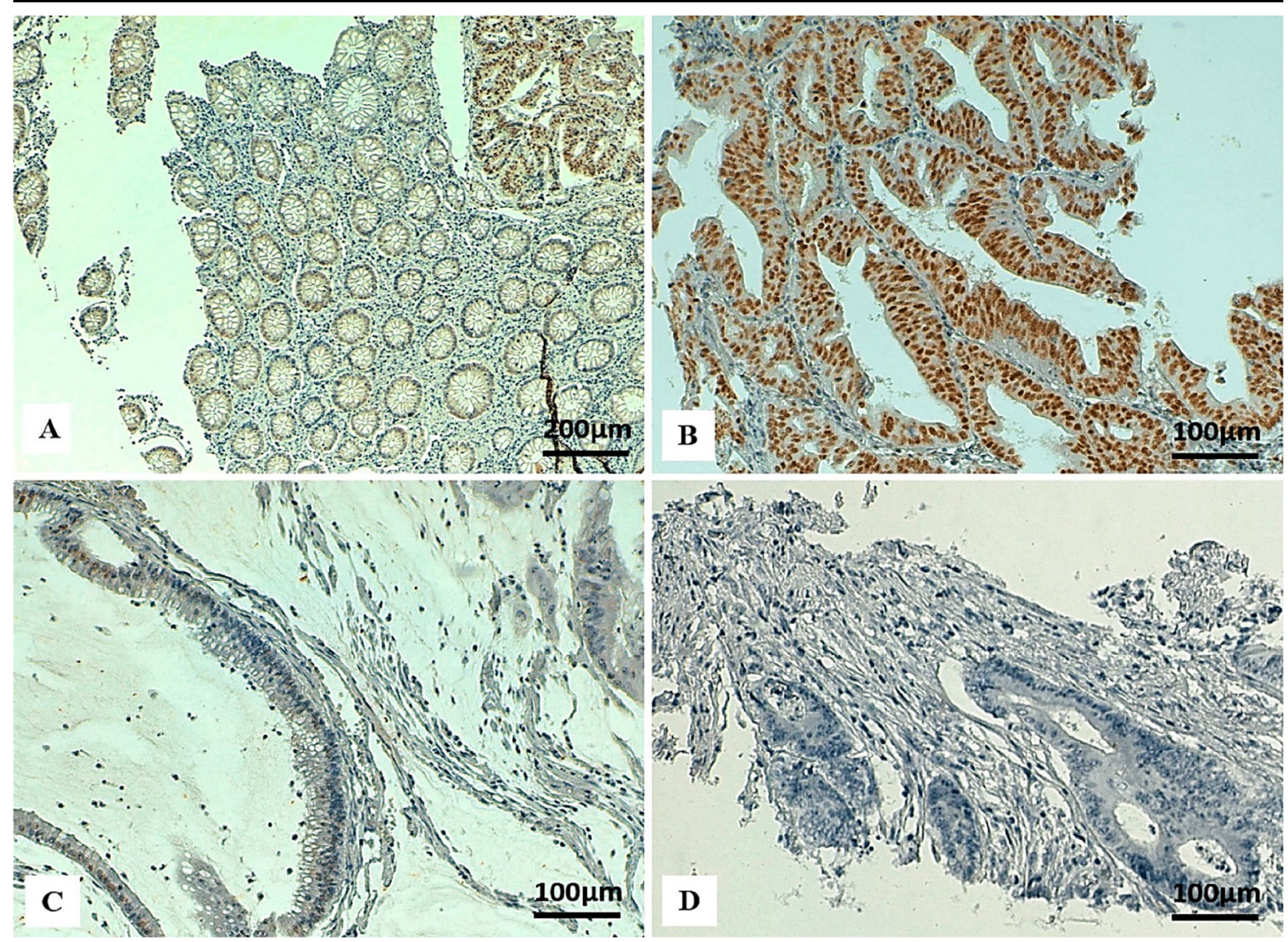

Figure 2 - Immunohistochemical staining for MSH2 in colon cancer: (A) Positive nuclear immunostaining for MSH2 in normal intestinal mucosa and in tumor cells (upper right corner) (×100); (B) Strong nuclear immunoreactivity for MSH2 in tumor cells $(\times 200)$; (C) Low intensity of nuclear immunostaining for MSH2 in tumor cells $(\times 200)$; (D) MSH2 loss in tumor cells (×200). MSH2: mutS homologue 2.
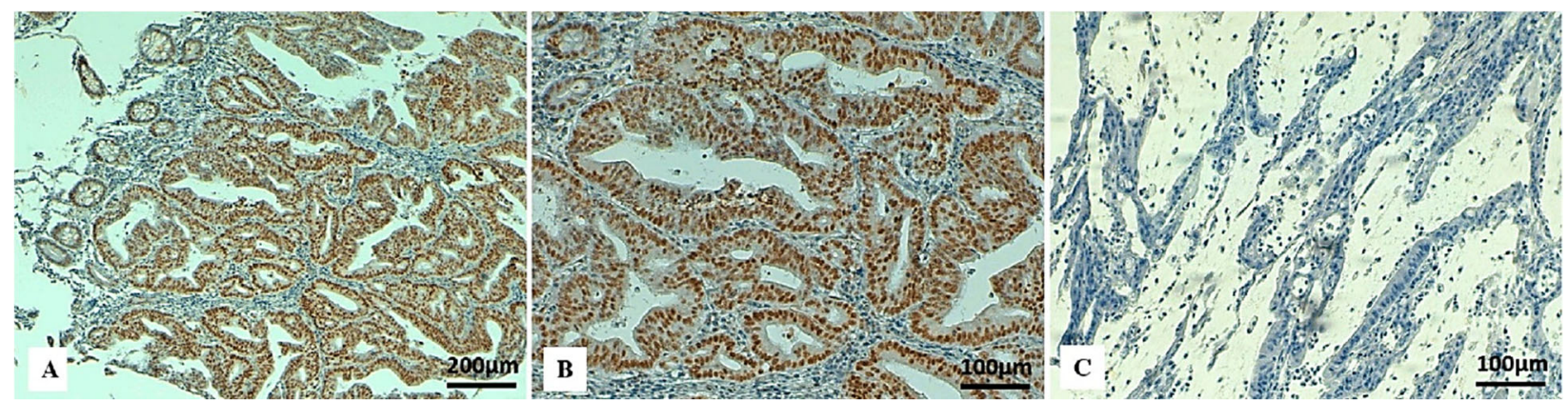

Figure 3 - Immunohistochemical staining for MSH6 in colon cancer: (A) Positive nuclear immunostaining for MSH2 in normal intestinal mucosa (left upper corner) and in tumor cells $(\times 100)$; (B) Strong positive nuclear immunoreactivity for MSH6 in tumor cells ( $\times 200)$; (C) Absence of nuclear immunostaining for MSH6 in tumor cells in a mucinous colon cancer (×200). MSH6: mutS homologue 6.

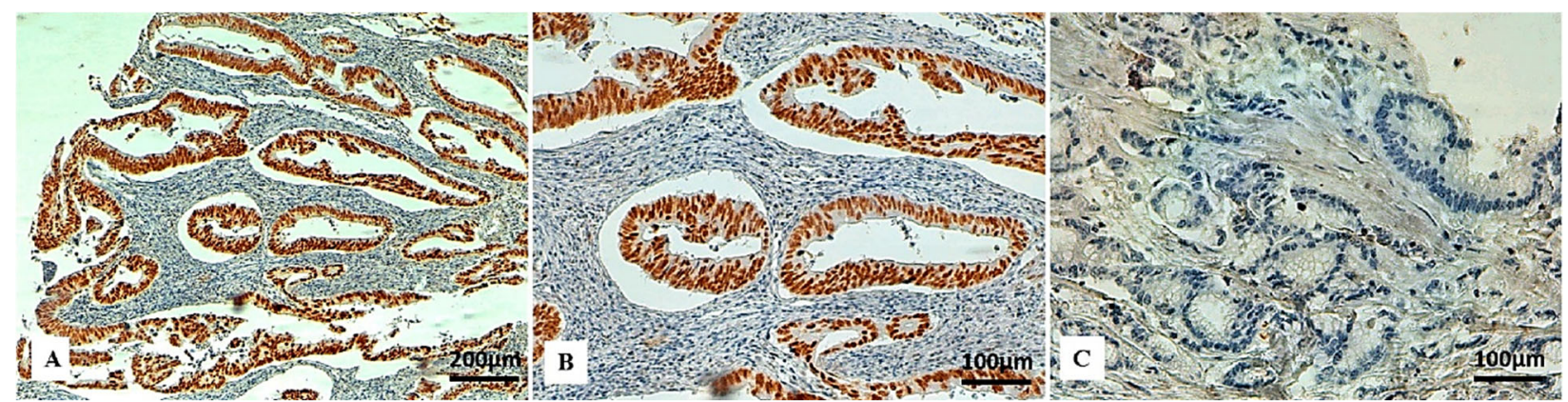

Figure 4 - Immunohistochemical staining for PMS2 in colon cancer: (A) Positive nuclear immunostaining for PMS2 in tumor cells ( $\times 100)$; (B) Strong positive nuclear immunostaining for PMS2 in tumor cells $(\times 200)$; (C) PMS2 loss in tumor cells $(\times 200)$. PMS2: Postmeiotic segregation increased 2. 
The relationship between individual loss of MMR protein immunoexpression and clinicopathological parameters is presented in Table 1. There was no significant relationship between isolated loss of MMR proteins and the clinicopathological features, such as: gender, tumor location, histological type, tumor stage, tumor grade, perineural invasion, vascular invasion, tumor necrosis, local invasion, peritumoral inflammatory infiltrate $(p>0.05)$ (Table 1). Relation between age and loss of MLH1 was at the limit of statistical significance $(p=0.066)$, tumors with loss of MLH1 being more frequent in young patients (under the age of 50). Correlation between lymph node metastasis $(\mathrm{pN})$ and individual MMR protein loss was not statistically significant, but near-marginal significance for MSH2 loss $(p=0.163)$ and PMS2 loss $(p=0.181)$.

No significant correlation was found between the individual expressions of MMR proteins and survival rate, but tumors with loss of MSH6 showed a tendency towards a better survival $(p=0.131)$ (Table 1$)$.

Table 1 - Association between individual loss of MMR protein immunoexpression and clinicopathological parameters

\begin{tabular}{|c|c|c|c|c|c|c|c|c|c|c|}
\hline & & $N(\%)$ & $\begin{array}{c}\text { MLH1 } \\
\text { loss } \\
\text { Mean [\%] }\end{array}$ & $p$-value & $\begin{array}{c}\text { MSH2 } \\
\text { loss } \\
\text { Mean [\%] }\end{array}$ & $p$-value & $\begin{array}{c}\text { MSH6 } \\
\text { loss } \\
\text { Mean [\%] }\end{array}$ & $p$-value & $\begin{array}{c}\text { PMS2 } \\
\text { loss } \\
\text { Mean [\%] }\end{array}$ & $p$-value \\
\hline \multirow{2}{*}{ Age [years] } & $<50$ & $5(16.12)$ & 42.66 & \multirow{2}{*}{0.066} & 45.21 & \multirow{2}{*}{0.654} & 49.38 & \multirow{2}{*}{0.984} & 37.81 & \multirow{2}{*}{0.830} \\
\hline & $>50$ & $26(83.87)$ & 18.13 & & 49.56 & & 50.33 & & 40.50 & \\
\hline \multirow{2}{*}{ Gender } & Female & $16(51.61)$ & 31.56 & \multirow{2}{*}{0.682} & 41.77 & \multirow{2}{*}{0.358} & 40.62 & \multirow{2}{*}{0.188} & 40.94 & \multirow{2}{*}{0.800} \\
\hline & Male & $15(48.39)$ & 29.97 & & 53.22 & & 59.67 & & 37.17 & \\
\hline \multirow{2}{*}{ Tumor site $^{1}$} & Right & $15(48.39)$ & 37.50 & \multirow{2}{*}{0.247} & 53.00 & \multirow{2}{*}{0.401} & 47.67 & \multirow{2}{*}{0.654} & 44.00 & \multirow{2}{*}{0.446} \\
\hline & Left & $16(51.61)$ & 24.50 & & 41.98 & & 51.88 & & 34.53 & \\
\hline \multirow{2}{*}{ Histological type ${ }^{2}$} & Intestinal & $21(67.74)$ & 33.54 & \multirow{2}{*}{0.262} & 47.98 & \multirow{2}{*}{1.00} & 58.57 & \multirow{2}{*}{0.356} & 41.96 & \multirow{2}{*}{0.493} \\
\hline & Mucinous & $10(32.26)$ & 28.53 & & 46.76 & & 42.65 & & 36.76 & \\
\hline \multirow{3}{*}{ Tumor grade $^{3}$} & G1 & $9(29.03)$ & 40.25 & \multirow{3}{*}{0.210} & 48.96 & \multirow{3}{*}{0.623} & 52.50 & \multirow{3}{*}{0.858} & 49.38 & \multirow{3}{*}{0.230} \\
\hline & G2 & $15(48.39)$ & 23.91 & & 42.50 & & 51.88 & & 31.25 & \\
\hline & G3 & $7(22.58)$ & 35.71 & & 56.43 & & 42.14 & & 45.36 & \\
\hline \multirow{3}{*}{$\mathrm{pN}$} & NO & $13(41.93)$ & 27.46 & \multirow{3}{*}{0.945} & 57.82 & & 39.20 & & 47.88 & \\
\hline & $N 1$ & $11(35.48)$ & 29.55 & & 47.73 & 0.163 & 35.63 & 0.238 & 28.64 & 0.181 \\
\hline & N2 & $7(22.58)$ & 38.93 & & 27.14 & & 31.43 & & 39.29 & \\
\hline & Absent & $24(77.41)$ & 29.69 & & 46.18 & & 53.13 & & 37.40 & \\
\hline Vascular emboll & Present & $7(22.58)$ & 34.57 & 1.00 & 51.19 & 0.129 & 38.57 & 0.317 & 45.00 & 0.473 \\
\hline & Absent & $24(77.41)$ & 29.69 & & 47.64 & & 49.17 & & 41.35 & \\
\hline Perıneural ınvasıon & Present & $7(22.58)$ & 34.57 & 1.00 & 46.19 & 0.945 & 52.14 & $0.8 / 2$ & 31.43 & 0.444 \\
\hline Lymphocytic & Absent & $17(54.83)$ & 29.85 & 0830 & 52.84 & & 48.24 & (9? & 38.38 & \\
\hline response & Present & $14(45.16)$ & 31.93 & 0.830 & 40.60 & 0.316 & 51.79 & 0.922 & 40.00 & 0.953 \\
\hline & Absent & $19(61.3)$ & 24.47 & 0367 & 46.75 & 0889 & 46.84 & 06 & 41.05 & 0857 \\
\hline Tumor necrosis & Present & $12(38.7)$ & 40.79 & 0.367 & 48.19 & 0.889 & 54.58 & 0.617 & 36.04 & 0.851 \\
\hline Metastasis & Absent & $28(90.32)$ & 32.12 & 826 & 43.45 & 5 & 49.29 & 07 & 37.23 & 0285 \\
\hline IVIetastasis & Present & $3(9.67)$ & 18.33 & & 63.33 & 0.55 & 55.00 & 0.171 & 56.67 & 0.285 \\
\hline & Absent & $29(93.54)$ & 28.26 & & 47.13 & & 50.17 & & 38.53 & \\
\hline Local invasion & Present & $2(6.45)$ & 67.50 & 0.129 & 50.00 & 1.00 & 45.00 & 0.727 & 47.50 & 0.520 \\
\hline & $I$ & $2(6.45)$ & 17.50 & & 55.00 & & 55.00 & & 45.00 & \\
\hline TNM & II & $9(29.03)$ & 33.00 & 0.995 & 50.74 & (202 & 58.89 & 0801 & 50.28 & \\
\hline INM staging [41] & III & $17(54.8)$ & 33.38 & 0.995 & 38.24 & $0 . \angle 0 Z$ & 43.53 & 0.801 & 29.41 & 0.226 \\
\hline & $I V$ & $3(9.67)$ & 18.33 & & 83.33 & & 55.00 & & 56.67 & \\
\hline & Present & $24(77.41)$ & 33.24 & & 44.20 & & 56.09 & & 38.59 & \\
\hline Survival status & Absent & $7(22.58)$ & 25.00 & & 52.86 & & 30.71 & 0.131 & 39.29 & 1.00 \\
\hline
\end{tabular}

MLH1: mutL homologue 1; MMR: Mismatch repair; MSH2: mutS homologue 2; MSH6: mutS homologue 6; N: No. of cases; PMS2: Postmeiotic segregation increased 2; TNM: Tumor, node, metastasis. ${ }^{1}$ Tumor site: right colon location - cecum, ascending colon; left colon location transverse segment to the sigmoid. ${ }^{2}$ Mucinous type: more than $25 \%$ mucus pools. ${ }^{3}$ Tumor grade: $\mathrm{G} 1$ - well differentiated; G2 - moderately differentiated; G3 - poorly differentiated. $p$-value $<0.05$ was considered statistically significant.

When comparing the relationship between MSS or MSI status and clinicopathological parameters (Table 2), we found a statistically significant correlation between MSI tumors and histological type $(p=0.03)$. Patients with MSI tumors had a high RR to develop mucinous tumors [RR: 2.045, 95\% confidence interval (CI): 0.966-3.033] than intestinal tumors [RR: $0.303,95 \%$ CI: $0.050-1.042]$. In this study, patients with deficient MMR proteins did not differ in terms of age, gender, tumor location, lymph node invasion, tumor differentiation grade, tumor stage, perineural invasion, vascular invasion, peritumoral inflammatory infiltrate, invasion of nearby organs (Table 2). No significant differences were identified in overall survival according to the MSI status $(p=0.199)$.

We found a positive aberrant cytoplasmatic expression for E-cadherin in $95.83 \%$ samples (Figure 5) and aberrant positive nuclear/cytoplasmatic beta-catenin immunostaining in all histological samples (Figure 6). There was no immunostaining for CDX2 in $25.80 \%$ cases (Figure 7).

IHC analysis of cell adhesion molecules (E-cadherin and beta-catenin), CDX2, clinicopathological features and survival rates is presented in Table 3 . 
Table 2 - Association between MMR status and clinicopathological characteristics

\begin{tabular}{|c|c|c|c|c|c|c|}
\hline & & $N(\%)$ & $\begin{array}{c}\text { MSS } \\
N(\%) \\
\end{array}$ & $\begin{array}{c}\text { MSI } \\
N(\%) \\
\end{array}$ & $p$-value & $\begin{array}{l}\text { Total } \\
\boldsymbol{N}(\%) \\
\end{array}$ \\
\hline \multirow{2}{*}{ Age [years] } & $<50$ & $5(16.12)$ & $3(9.67)$ & $2(6.45)$ & \multirow{2}{*}{$1.000^{* *}$} & $5(16.12)$ \\
\hline & $>50$ & $26(83.87)$ & $20(54.8)$ & $6(29)$ & & $26(83.9)$ \\
\hline \multirow{2}{*}{ Gender } & Female & $16(51.61)$ & $11(35.48)$ & $5(16.12)$ & \multirow{2}{*}{$0.809^{*}$} & $16(51.6)$ \\
\hline & Male & $15(48.39)$ & $12(38.70)$ & $3(9.67)$ & & $15(48.39)$ \\
\hline \multirow{2}{*}{ Tumor site $^{1}$} & Right & 15 (48.39) & $11(35.48)$ & $4(12.90)$ & \multirow{2}{*}{$0.909^{*}$} & 15(48.39) \\
\hline & Left & $16(51.61)$ & $12(38.70)$ & $4(12.90)$ & & $16(51.61)$ \\
\hline \multirow{2}{*}{ Histological type ${ }^{2}$} & Intestinal & $21(67.74)$ & $18(58.06)$ & $3(9.67)$ & \multirow{2}{*}{$0.03^{*}$} & $21(67.74)$ \\
\hline & Mucinous & $10(32.26)$ & $5(16.13)$ & $5(16.13)$ & & $10(32.26)$ \\
\hline \multirow{3}{*}{ Tumor grade ${ }^{3}$} & G1 & $9(29.03)$ & $8(25.80)$ & $1(3.2)$ & \multirow{3}{*}{$0.288^{*}$} & $9(29.03)$ \\
\hline & G2 & $15(48.38)$ & $11(35.48)$ & $4(12.90)$ & & $15(48.38)$ \\
\hline & G3 & $7(22.58)$ & $4(12.90)$ & $3(9.67)$ & & $7(22.58)$ \\
\hline \multirow{3}{*}{$\mathrm{pN}$} & NO & $13(41.93)$ & $10(32.25)$ & $3(9.67)$ & \multirow{3}{*}{$0.455^{*}$} & $13(41.93)$ \\
\hline & N1 & $11(35.48)$ & $9(29.03)$ & $2(6.45)$ & & $11(35.48)$ \\
\hline & N2 & $7(22.58)$ & $4(12.90)$ & $3(9.67)$ & & $7(22.58)$ \\
\hline \multirow{2}{*}{ Venous emboli } & Absent & $24(77.41)$ & $17(54.83)$ & $7(22.58)$ & \multirow{2}{*}{$0.664^{*}$} & $24(77.41)$ \\
\hline & Present & $7(22.58)$ & $6(19.35)$ & $1(3.22)$ & & $7(22.58)$ \\
\hline \multirow{2}{*}{ Perineural invasion } & Absent & $24(77.41)$ & $17(54.83)$ & $7(22.58)$ & \multirow{2}{*}{$0.664^{*}$} & $24(77.41)$ \\
\hline & Present & $7(22.58)$ & $6(19.35)$ & $1(3.22)$ & & $7(22.58)$ \\
\hline \multirow{2}{*}{ Lymphocytic response } & Absent & $17(54.83)$ & $14(45.16)$ & $3(9.67)$ & \multirow{2}{*}{$0.436^{*}$} & $17(54.81)$ \\
\hline & Present & $14(45.16)$ & $9(29.03)$ & $5(16.12)$ & & $14(45.16)$ \\
\hline \multirow{2}{*}{ Tumor necrosis } & Absent & $19(61.3)$ & $15(48.38)$ & $4(12.90)$ & \multirow{2}{*}{$0.567^{*}$} & $19(61.3)$ \\
\hline & Present & $12(38.7)$ & $8(25.80)$ & $4(12.90)$ & & $12(38.7)$ \\
\hline \multirow{2}{*}{ Metastasis } & Absent & $28(90.32)$ & $20(64.51)$ & $8(25.80)$ & \multirow{2}{*}{$0.535^{\star \star}$} & $28(90.32)$ \\
\hline & Present & $3(9.67)$ & $3(9.67)$ & $0(0.0)$ & & $3(9.67)$ \\
\hline \multirow{2}{*}{ Invasion in nearby organs } & Absent & $29(93.54)$ & $21(67.74)$ & $8(25.80)$ & \multirow{2}{*}{$0.527^{* *}$} & $29(93.5)$ \\
\hline & Present & $2(6.45)$ & $2(6.45)$ & $0(0)$ & & $2(6.45)$ \\
\hline \multirow{4}{*}{ TNM staging [41] } & 1 & $2(6.45)$ & $2(6.45)$ & $0(0)$ & \multirow{4}{*}{$0.597^{*}$} & $2(6.45)$ \\
\hline & II & $9(29.03)$ & $6(19.35)$ & $3(9.67)$ & & $9(29.0)$ \\
\hline & III & $17(54.8)$ & $11(35.48)$ & $5(16.12)$ & & $17(54.8)$ \\
\hline & IV & $3(9.7)$ & $3(9.67)$ & $0(0)$ & & $3(9.7)$ \\
\hline \multirow{2}{*}{ Survival status } & Living & $24(77.41)$ & $19(61.29)$ & $5(16.12)$ & \multirow{2}{*}{$0.199^{*}$} & $24(77.41)$ \\
\hline & Deceased & $7(22.58)$ & $4(12.90)$ & $3(9.67)$ & & $7(22.58)$ \\
\hline
\end{tabular}

MMR: Mismatch repair; MSI: Microsatellite instability; MSS: Microsatellite stable; $N$ : No. of cases; TNM: Tumor, node, metastasis. ${ }^{1}$ Tumor site: right colon location - cecum, ascending colon; left colon location - transverse segment to the sigmoid. ${ }^{2}$ Mucinous type: more than $25 \%$ mucus pools. ${ }^{3}$ Tumor grade: G1 - well differentiated; G2 - moderately differentiated; G3 - poorly differentiated. $p$-value $<0.05$ was considered statistically significant. ${ }^{*}$ Chi-squared test; ${ }^{*}$ Fisher's exact test.
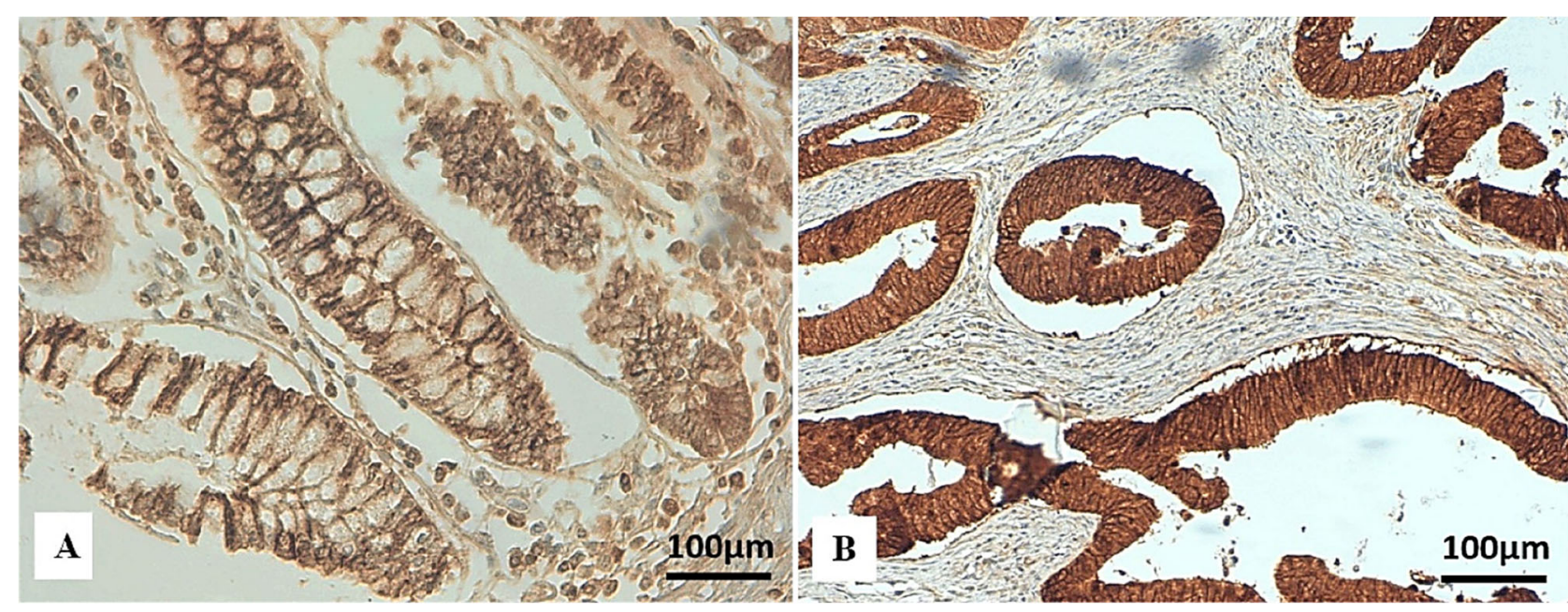

Figure 5 - Immunohistochemical staining for E-cadherin in colon cancer: (A) Normal membranous immunoexpression of E-cadherin in normal colon mucosa ( $\times 200)$; (B) Strong cytoplasmatic and membranous immunoexpression of E-cadherin in tumor cells $(\times 200)$. 
Assessment of mismatch repair deficiency, CDX2, beta-catenin and E-cadherin expression in colon cancer: molecular... 721

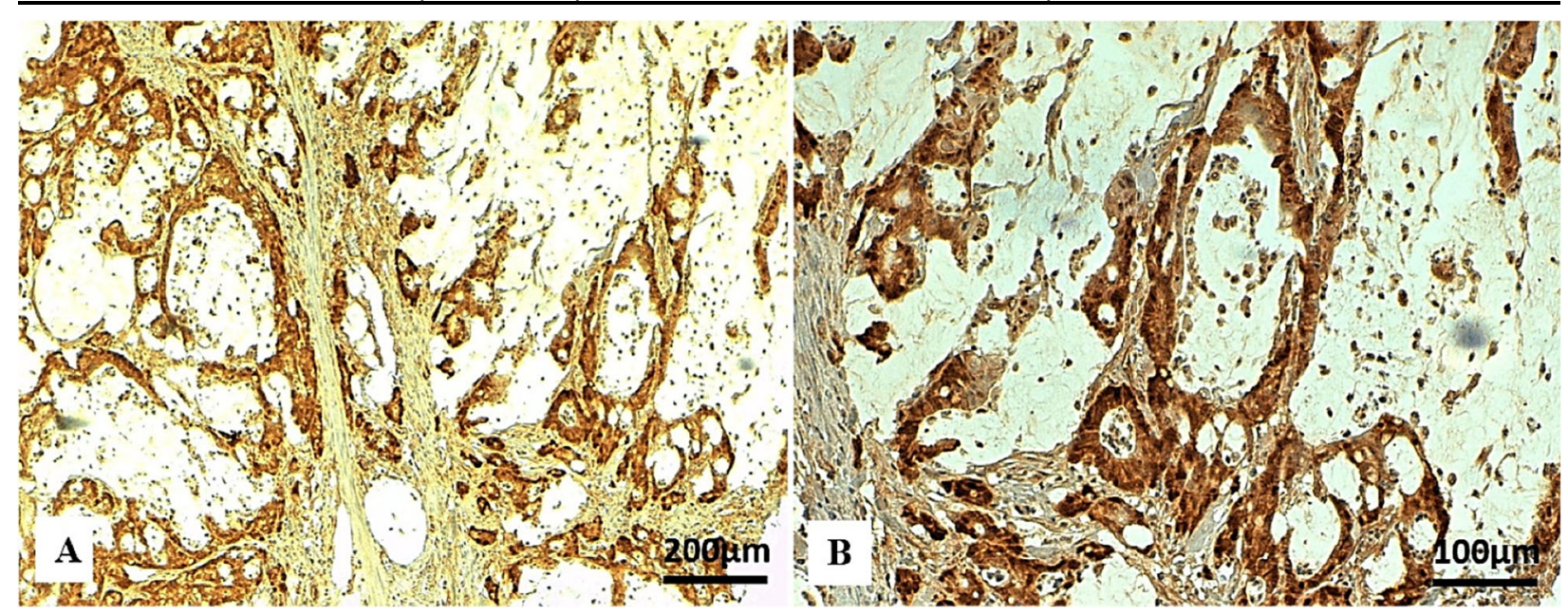

Figure 6 - Immunohistochemical staining for beta-catenin in colon cancer: (A) Diffuse beta-catenin immunopositivity in a mucinous colon adenocarcinoma $(\times 100)$; (B) Strong nuclear immunostaining for beta-catenin in tumor cells $(\times 200)$.

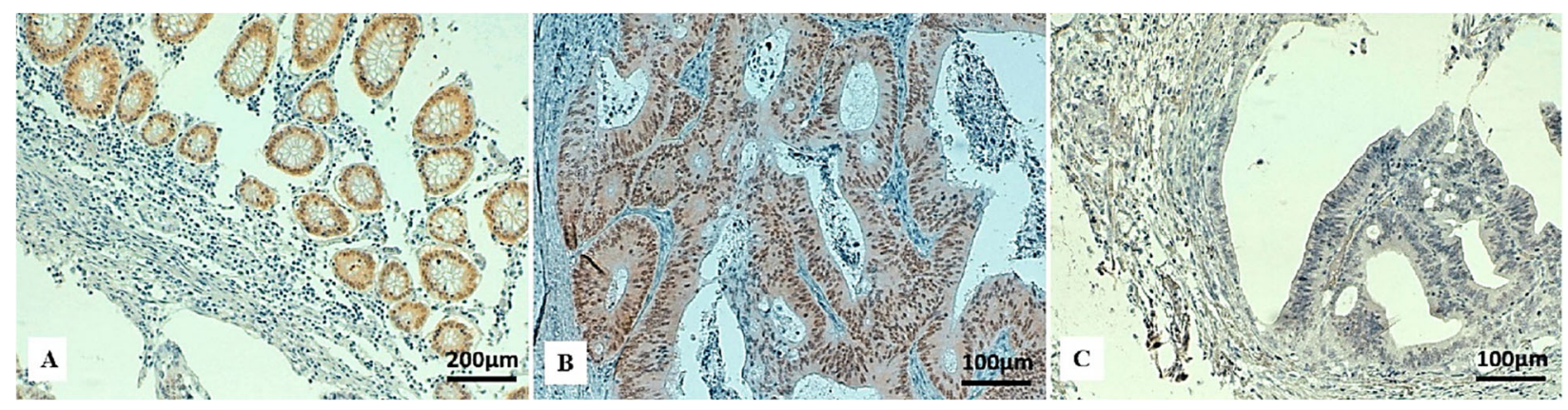

Figure 7 - Immunohistochemical staining for CDX2 in colon cancer: (A) Positive nuclear immunostaining for CDX2 in normal intestinal mucosa $(\times 100)$; (B) Strong positive nuclear immunoreactivity for CDX2 in tumor cells $(\times 200)$; (C) Absence of nuclear immunostaining for CDX2 in tumor cells (×200). CDX2: Caudal-type homeobox 2.

Table 3 - Interrelation between beta-catenin, E-cadherin, CDX2 protein immunoexpression and clinicopathological parameters

\begin{tabular}{|c|c|c|c|c|c|c|c|c|c|c|}
\hline & & $N(\%)$ & $\begin{array}{c}\text { Beta- } \\
\text { catenin } \\
\text { Mean [\%] }\end{array}$ & $p$-value & $\begin{array}{c}\text { E-cadherin } \\
\text { Mean [\%] }\end{array}$ & $p$-value & $\begin{array}{c}\text { CDX2 } \\
\text { positive } \\
\text { Mean [\%] }\end{array}$ & $p$-value & $\begin{array}{l}\text { CDX2 } \\
\text { loss } \\
N(\%)\end{array}$ & $p$-value \\
\hline \multirow{2}{*}{ Age [years] } & $<50$ & $5(16.12)$ & 93.44 & \multirow{2}{*}{0.654} & 73.44 & \multirow{2}{*}{0.830} & 32.34 & \multirow{2}{*}{0.740} & $3(9.67)$ & \multirow{2}{*}{0.43} \\
\hline & $>50$ & $26(83.87)$ & 93.00 & & 72.67 & & 31.67 & & $5(16.12)$ & \\
\hline \multirow{2}{*}{ Gender } & Female & $16(51.6)$ & 92.81 & \multirow{2}{*}{1.00} & 75.94 & \multirow{2}{*}{0.446} & 37.03 & \multirow{2}{*}{0.800} & $3(9.67)$ & \multirow{2}{*}{0.43} \\
\hline & Male & $15(48.4)$ & 93.67 & & 70.00 & & 26.67 & & $5(16.12)$ & \\
\hline \multirow{2}{*}{ Tumor site $^{1}$} & Right & $15(48.4)$ & 95.00 & \multirow{2}{*}{0.299} & 76.67 & \multirow{2}{*}{0.599} & 40.00 & \multirow{2}{*}{0.151} & 2 & \multirow{2}{*}{0.20} \\
\hline & Left & $16(51.6)$ & 91.56 & & 69.69 & & 24.53 & & 6 & \\
\hline \multirow{2}{*}{$\begin{array}{l}\text { Histological } \\
\text { type }^{2}\end{array}$} & Intestinal & $21(67.74)$ & 94.29 & \multirow{2}{*}{0.681} & 70.00 & \multirow{2}{*}{0.493} & 31.25 & \multirow{2}{*}{0.653} & $3(9.67)$ & \multirow{2}{*}{0.69} \\
\hline & Mucinous & $10(32.26)$ & 92.35 & & 75.59 & & 32.65 & & $5(16.12)$ & \\
\hline \multirow{3}{*}{ Tumor grade ${ }^{3}$} & G1 & $9(29.03)$ & 92.50 & \multirow{3}{*}{0.237} & 83.13 & \multirow{3}{*}{0.018} & 35.31 & & $3(9.67)$ & \multirow{3}{*}{0.58} \\
\hline & G2 & $15(48.38)$ & 91.88 & & 68.12 & & 25.63 & 0.353 & 4 & \\
\hline & G3 & $7(22.58)$ & 97.14 & & 72.86 & & 42.86 & & 1 & \\
\hline \multirow{3}{*}{$\mathrm{pN}$} & NO & $13(41.9)$ & 94.23 & \multirow{3}{*}{0.046} & 73.08 & \multirow{3}{*}{0.277} & 34.04 & \multirow{3}{*}{0.837} & 4 & \multirow{3}{*}{0.24} \\
\hline & N1 & $11(35.48)$ & 89.09 & & 68.64 & & 33.64 & & 1 & \\
\hline & N2 & $7(22.6)$ & 97.86 & & 80.00 & & 25.71 & & $3(9.67)$ & \\
\hline \multirow{2}{*}{ Venous emboli } & Absent & $24(77.4)$ & 92.71 & \multirow{2}{*}{0.563} & 72.08 & & 35.52 & & 6 & \\
\hline & Present & $7(22.6)$ & 95.00 & & 76.43 & 0.473 & 20.00 & 0.532 & 2 & 1.00 \\
\hline Perineural & Absent & 24 (77.41) & 94.79 & & 73.54 & & 35.31 & & $5(16.12)$ & \\
\hline invasion & Present & $7(22.6)$ & 87.86 & 0.068 & 71.43 & 0.661 & 20.71 & 0.235 & $3(9.67)$ & 0.33 \\
\hline Lymphocytic & Absent & $17(54.8)$ & 93.24 & & 70.00 & & 35.74 & & $3(9.67)$ & \\
\hline response & Present & $14(45.2)$ & 93.21 & 0.769 & 76.79 & 0.399 & 27.50 & 0.316 & $5(16.12)$ & 0.41 \\
\hline Tumor necrosis & Absent & $19(61.3)$ & 92.11 & 0389 & 76.05 & & 39.61 & & 4 & 067 \\
\hline Tumor necrosis & Present & $12(38.7)$ & 95.00 & 0.389 & 68.33 & 0.389 & 20.00 & 0.220 & 4 & 0.67 \\
\hline Metastasis & Absent & $28(90.32)$ & 93.04 & 0777 & 72.32 & & 30.80 & 0285 & 8 & 055 \\
\hline Metastasıs & Present & $3(9.78)$ & 95.00 & 0.176 & 80.00 & 0.422 & 43.33 & 0.285 & 0 & 0.55 \\
\hline
\end{tabular}




\begin{tabular}{|c|c|c|c|c|c|c|c|c|c|c|}
\hline & & $N(\%)$ & $\begin{array}{c}\text { Beta- } \\
\text { catenin } \\
\text { Mean [\%] }\end{array}$ & $p$-value & $\begin{array}{l}\text { E-cadherin } \\
\text { Mean [\%] }\end{array}$ & $p$-value & $\begin{array}{c}\text { CDX2 } \\
\text { positive } \\
\text { Mean [\%] } \\
\end{array}$ & $p$-value & $\begin{array}{l}\text { CDX2 } \\
\text { loss } \\
N(\%) \\
\end{array}$ & $p$-value \\
\hline \multirow{2}{*}{$\begin{array}{c}\text { Invasion in } \\
\text { nearby organs }\end{array}$} & Absent & $29(93.5)$ & 92.76 & \multirow{2}{*}{0.211} & 72.41 & \multirow{2}{*}{0.310} & 32.16 & \multirow{2}{*}{0.619} & 8 & \multirow{2}{*}{1.00} \\
\hline & Present & $2(6.5)$ & 100.00 & & 82.50 & & 30.00 & & 0 & \\
\hline \multirow{4}{*}{$\begin{array}{c}\text { TNM staging } \\
{[41]}\end{array}$} & $I$ & $2(6.5)$ & 92.50 & \multirow{4}{*}{0.932} & 75.00 & \multirow{4}{*}{0.816} & 61.25 & \multirow{4}{*}{0.238} & 0 & \multirow{4}{*}{0.33} \\
\hline & II & $9(29)$ & 94.44 & & 70.56 & & 23.89 & & 4 & \\
\hline & III & $17(54.83)$ & 92.35 & & 72.94 & & 30.88 & & 4 & \\
\hline & IV & $3(9.67)$ & 95.00 & & 80.00 & & 43.33 & & 0 & \\
\hline \multirow{2}{*}{ Survival status } & Living & $24(77.41)$ & 93.26 & \multirow{2}{*}{0.924} & 70.87 & \multirow{2}{*}{0.288} & 31.41 & \multirow{2}{*}{0.924} & 6 & \multirow{2}{*}{1.00} \\
\hline & Deceased & $7(22.6)$ & 94.29 & & 80.71 & & 34.29 & & 2 & \\
\hline
\end{tabular}

CDX2: Caudal-type homeobox 2; N: No. of cases; TNM: Tumor, node, metastasis. ${ }^{1}$ Tumor site: right colon location - cecum, ascending colon; left colon location - transverse segment to the sigmoid. ${ }^{2}$ Mucinous type: more than $25 \%$ mucus pools. ${ }^{3}$ Tumor grade: G1 - well differentiated; G2 - moderately differentiated; G3 - poorly differentiated. $p$-value $<0.05$ was considered statistically significant.

Statistically significant correlation was noticed between the percentage of positive cytoplasmatic E-cadherin cells and the tumor differentiation degree ( $p=0.018$; MannWhitney $U$-test for independent samples), tumors with low differentiation degree displaying aberrant E-cadherin expression. Moreover, there was a statistically significant correlation between the intensity of beta-catenin immunoexpression and lymph node invasion $(p=0.046$; MannWhitney $U$-test for independent samples), tumors with lymph node metastasis having a strong protein expression.

No significant association between CDX2 expression and tumor clinical or pathological features was found $(p>0.05)$ (Table 3).
No significant differences were identified in overall survival according to E-cadherin, beta-catenin or CDX2 immunoexpression (Table 3).

Moreover, we analyzed the correlation between individual loss of MMR protein expression and CDX2 loss (Table 4). Individual MLH1 loss was strongly correlated to PMS2 loss $(p=0.006)$ and to MSH2 loss $(p=0.023)$; MSH2 loss was significant associated to MSH6 loss $(p=0.011)$ and PMS2 loss was significantly correlated to CDX2 loss $(p=0.03)$.

Table 4 - Correlation between individual loss of MMR proteins and CDX2 loss

\begin{tabular}{|c|c|c|c|c|c|c|c|}
\hline & & & CDX2 loss & MLH1 loss & MSH2 loss & MSH6 loss & PMS2 loss \\
\hline \multirow{15}{*}{$\begin{array}{l}\text { Spearman's } \\
\text { rho }\end{array}$} & \multirow{3}{*}{$\begin{array}{c}\text { CDX2 } \\
\text { loss }\end{array}$} & Correlation coefficient & 1.000 & .332 & .283 & .093 & $.391^{*}$ \\
\hline & & $\overline{p \text {-value }}$ & . & .068 & .123 & .619 & .030 \\
\hline & & $\bar{N}$ & 31 & 31 & 31 & 31 & 31 \\
\hline & \multirow{3}{*}{$\begin{array}{c}\text { MLH1 } \\
\text { loss }\end{array}$} & Correlation coefficient & .332 & 1.000 & $.408^{*}$ & .258 & $.479^{\star *}$ \\
\hline & & $p$-value & .068 &. & .023 & .161 & .006 \\
\hline & & $N$ & 31 & 31 & 31 & 31 & 31 \\
\hline & \multirow{3}{*}{$\begin{array}{c}\text { MSH2 } \\
\text { loss }\end{array}$} & Correlation coefficient & .283 & $.408^{*}$ & 1.000 & $.450^{*}$ & .183 \\
\hline & & $p$-value & .123 & .023 &. & .011 & .324 \\
\hline & & $N$ & 31 & 31 & 31 & 31 & 31 \\
\hline & \multirow{3}{*}{$\begin{array}{c}\text { MSH6 } \\
\text { loss }\end{array}$} & Correlation coefficient & .093 & .258 & $.450^{*}$ & 1.000 & .134 \\
\hline & & $p$-value & .619 & .161 & .011 & . & .473 \\
\hline & & $\bar{N}$ & 31 & 31 & 31 & 31 & 31 \\
\hline & \multirow{3}{*}{$\begin{array}{l}\text { PMS2 } \\
\text { loss }\end{array}$} & Correlation coefficient & $.391^{*}$ & $.479^{\star *}$ & .183 & .134 & 1.000 \\
\hline & & $\overline{p \text {-value }}$ & .030 & .006 & .324 & .473 &. \\
\hline & & $N$ & 31 & 31 & 31 & 31 & 31 \\
\hline
\end{tabular}

CDX2: Caudal-type homeobox 2; MLH1: mutL homologue 1; MMR: Mismatch repair; MSH2: mutS homologue 2; MSH6: mutS homologue 6; $N$ : No. of cases; PMS2: Postmeiotic segregation increased 2. ${ }^{\star}$ Correlation is significant at the 0.05 level $\left(2\right.$-tailed). ${ }^{* *}$ Correlation is significant at the 0.01 level (2-tailed).

\section{ค Discussions}

$\mathrm{CRC}$ is a heterogeneous disease with different clinical, pathological, and molecular features, even within the same TNM stage, probably because of different molecular mechanisms involved in colon tumorigenesis $[4,6]$. Many existing studies have examined the relationship between different biological markers involved in colon cancer progression, but the results are controversial.

MSI or the deficiency of MMR proteins is one of the molecular pathways of colorectal tumorigenesis and characterizes approximately $15-20 \%$ of CRCs $[3,4,43$, 44].

Our study aimed to evaluate the correlation between the clinicopathological features in colon cancer and the IHC expression of different biomarkers, such as: MMR proteins (MLH1, MSH2, MSH6, PMS2), CDX2 and adhesion molecules (E-cadherin, beta-catenin). Additionally, we investigated the association between the selected biomarkers and overall survival.

We detected a dMMR proteins in $26.81 \%$ cases and the most frequently noticed protein loss was MLH1 (75\%). These results are similar to those reported by other authors $[10,11,39,45]$.

In our study, most of the cases with MLH1 loss also comprised PMS2 loss (5/6 patients), which is in concordance with the results of other studies, that claimed that MLH1 and PMS2 are often lost together, and the defect is caused 
by mutation in the $M L H 1$ gene, which is essential for stabilization of PMS2 [25, 26, 46]. The PMS2 loss was detected in $62.5 \%$ of cases, similar with the proportion reported by other authors [39].

It was interesting the fact that, in the present study, the tumors with PMS2 loss, together with tumors with MSH6 loss, covered all the dMMR tumors. Other authors reported similar results [39, 45, 47], suggesting that probably the double panel (PMS2 and MSH6) is enough to detect the dMMR status, instead of using the quadruple panel.

Our study did not find any significant relationship between individual loss of MMR proteins (MLH1, MSH2, MSH6, PMS2) and the clinicopathological features, such as: gender, tumor location, histological type, tumor stage, tumor grade, invasion, necrosis, etc. We found that tumors with loss of MLH1 were more frequent in young patients (under the age of 50), even if the difference was only approaching the significance level $(p=0.06)$. In the current study, tumors with MSH2 loss and PMS2 loss showed high propensity for lymph node metastasis but the results were not statistically significant $(p=0.161$ and $p=0.181$, respectively).

Previous studies have shown controversial results. Some authors suggest that isolated loss of MLH1 or MSH2 is associated with right colon tumor location [41], poorly differentiated or mucinous differentiation [41, 48, 49], lymph node metastasis [49], lymphovascular invasion [41], peritumoral inflammatory infiltrate [41].

When comparing the two patient subgroups: MSI vs. MSS tumors and their association with clinicopathological features, the MSI status in the present study was statistically significantly correlated with mucinous histological type $(p=0.003)$. Similar results were reported by some authors [50-52]. Our data did not reveal other particular clinicopathological features for MSI tumors. Several authors have found a positive association between MSI status and younger age [24, 50], gender [10], right location [10, 24, 50-53], poor differentiation [24, 39], lymph node invasion $[39,50]$. On the other hand, some other studies did not show any significant correlation between MSI status and age [10, 53], gender [24, 39, 53], histological type [24], lymph node invasion [24, 39], tumor differentiation grade [53], tumor stage [24, $39,53]$, perineural invasion, [10, 24, 39], vascular [10, 24, 39], peritumoral inflammatory infiltrate [24].

The present study revealed that individual MLH1 loss was strongly correlated to PMS2 loss $(p=0.006)$ and MSH2 loss $(p=0.023)$. These findings are similar to those reported by other authors [25, 46], who also revealed that PMS2 loss was often associated with MLH1 loss. Moreover, MSH2 loss was significant associated to MSH6 loss $(p=0.011)$. This can be explained by the fact that MMR proteins form dimers and work in pairs, MLH1/ PMS2 and MSH2/MSH6 [12, 24, 46], therefore loss of MLH1 or MLH2 induces loss of PMS2, respectively MSH6. However, loss of PMS2, respectively MSH6, is not always associated with MLH1 respectively MLH2 loss, maybe because of other MMR proteins (e.g., MSH3) which bind and stabilize MLH1 or MSH2 [46].

Our data showed better survival for patients with MSI tumors, but the difference was not statistically significant $(p=0.199)$. We also found that tumors with individual loss of MSH6 showed tendency towards a better survival $(p=0.131)$. Since in our study mucinous histological type has been associated with MSI status, and mucinous tumors usually tend to be associated with poor prognosis [52], we could suggest that there is a subgroup of mucinous colon tumors with MSI status that may have better outcomes [52].

There is a considerable body of literature on the relationship between the overall survival and MSI status; several systematic reviews and meta-analysis have shown an improved overall survival in patients with MMR deficiency [3, 54, 55]. The meta-analysis conducted by Guastadisegni et al. [54] involving 12782 patients revealed a significantly improved prognosis for MSI tumors [odds ratio (OR): $0.58,95 \% \mathrm{CI}$ : $0.47-0.72, p<0.0001]$. Even so, other authors did not find any significant correlation between MMR deficient status and overall survival [3, 13, 49].

E-cadherin is a transmembrane glycoprotein normally expressed in the basolateral membrane of epithelial cells (adherens junctions), being involved in growth and development in the early human organogenesis, as well as in cell-to-cell interactions $[30,56,57]$. In pathological conditions, it has been associated with loss of epithelial differentiation, EMT, tumor progression, invasion, metastasis, because of loss of cell adhesiveness [30, 31].

Beta-catenin is a structural component of cadherinbased adherens junctions and an important component of the Wnt signal cascade $[33,58,59]$, playing a role in the control of cell proliferation or cell death [60]. Betacatenin has been reported to be implicated in carcinogenesis, activation of this pathway causing an aberrant accumulation of nuclear/cytoplasmatic beta-catenin and reduced membranous expression [33, 58, 59].

Immunohistochemistry is an effective technique to identify reduced membranous expression levels of adhesion molecules, aberrant E-cadherin, and beta-catenin expression and Wnt pathway activation.

In the current study, we detected a high percentage of aberrant E-cadherin and beta-catenin immunoexpression in the tumor cells $(95-100 \%)$. Other authors have also reported high proportion (80-100\%) of aberrant E-cadherin or beta-catenin expression in colon cancer cells [61-64].

We found a statistically significant correlation between the percentage of aberrant cytoplasmatic E-cadherin cells and the tumor differentiation degree $(p=0.018)$, which is consistent with the results of other studies [28, 31, 39, $56,61,62]$, where aberrant or decreased membranous E-cadherin immunoexpression was associated with a low degree of tumor differentiation. These findings could be explained in part by the fact that during the EMT, the dissolution of the E-cadherin-mediated adherens junction causes loss of E-cadherin normal immunoexpression [31, 65].

Furthermore, in our study, a statistically significant correlation was detected between the intensity of nuclear beta-catenin immunoexpression and lymph node invasion $(p=0.046)$, tumors with lymph node metastasis having a strong beta-catenin nuclear expression. Our data are in accordance with the results published by other authors which indicate that high nuclear beta-catenin expression has a high incidence of lymph node metastasis and shorter overall survival $[66,67]$. 
In our group of patients, there was no significant correlation between E-cadherin or beta-catenin aberrant expression and other different clinicopathological parameters (gender, age, tumor location, histological type, tumor stage, perineural invasion, vascular invasion, peritumoral inflammatory infiltrate) our results being similar to other published studies $[61,62]$. The present study did not find any statistically significant correlation between aberrant adhesion molecules expression and local invasion or metastasis. This was a surprising result, considering the role of these molecules in cell-to-cell interactions, and can be explained by the fact that in cancer progression, alteration of adhesion proteins can be associated with alterations of other molecules [68]. Ahmad \& HahnStromberg [69] also reported no statistical association between E-cadherin and beta-catenin immunoexpression and metastasis in colon cancer, while other authors [61, $62,64]$ proved that E-cadherin or beta-catenin aberrant expression led to a risk of aggressive biological behavior.

Previous research has reported divergent results concerning the prognostic value of E-cadherin and betacatenin in colon tumors $[56,61,69,70]$. In the current study, we did not identify any significant effect of E-cadherin or beta-catenin on the survival rate. Some groups have reported similar results $[56,71]$, while others have found that loss of normal immunoreactivity of these adhesion proteins may be a predictor of poor survival in colon cancer $[62,64,72]$, as well as in other tumors: breast cancer [73], hepatocellular carcinoma [74], bladder cancer [75], prostate cancer [76], cervical cancer [77].

CDX2 is an intestinal transcription factor, normally expressed in the nucleus of enterocytes [35, 36], that plays an essential role in intestinal development and differentiation during embryonic life [35, 78, 79]. In pathological conditions, CDX2 is an important marker for intestinal adenocarcinomas [36, 37, 39]. Immunohistochemistry identifies nuclear CDX2 expression in both normal and neoplastic colonic mucosa.

In the current study, loss of nuclear CDX2 expression was identified in $25.80 \%$ cases, similar to other previous reports [36, 80, 81]. No significant correlation between the loss of CDX2 immunoexpression and any of the clinicopathological features was found in the present study $(p>0.05)$. Our findings are similar to those reported by Altintas et al. [82], but the literature review shows divergent results concerning the relationship between the CDX2 loss and clinicopathological features. Some authors reported a significant correlation between CDX2 loss and female gender [80, 81], right-sided tumor location [79, 80, 83], high-grade tumors [36, 39, 79, 81, 83], advanced tumor stage [36, 39, 79, 81, 83], increased number of tumor-infiltrating lymphocytes [79], mucin production [79].

In our study, PMS2 loss was significantly correlated to $\mathrm{CDX} 2$ loss $(p=0.03)$. Similar results were reported by Sayar et al. [39]. Although there are studies that suggested a relationship between CDX2 loss and MMR protein deficiency [79, 83], other authors [84] showed no association between MSI status and CDX2 loss.

Our data did not reveal any significant impact of loss of CDX2 expression on overall survival, which is in accordance with the results of other studies [78, 81, 82, 85]. In contrast, several studies suggested that CDX2 loss was independently associated with worse overall survival [79] and progression-free survival [79, 86, 87]. In a recently published study, Ryan et al. [88] proved that CDX2 loss expression had a poor prognosis only in patients with MSS CRC, and patients with MSI CDX2 loss had a low risk to develop distant metastases. Therefore, maybe CDX2 loss is a worse prognostic factor only in the subgroup of patients with MSS CRC $[87,88]$.

Despite the numerous studies, the role of CDX2 in colorectal carcinogenesis is poorly understood and it might differ in relation with other proteins involved in tumor progression.

\section{Study limitations}

Limitations of our study can be related to some reasons: the small number of patients enrolled, the tumor heterogeneity, different types of antibodies used, various scoring systems that identify the aberrant antigen immunoexpression, etc.

\section{Conclusions}

Despite decades of research, the molecular analysis of biological markers for colon cancer continues to be a very interesting debate topic. The results of this study indicate that MSI status was statistically significantly correlated with mucinous histological type, but not with other pathological features or with survival rate. Our data indicate that MLH1 and PMS2 are often lost together. Moreover, tumors with PMS2 loss, together with tumors with MSH6 loss, covered all the dMMR tumors, suggesting that the double panel (PMS2 and MSH6) might be enough for detecting the MSI status. Our study demonstrated that the adhesion molecules (E-cadherin, beta-catenin), hallmarks of EMT, were positively associated with tumor differentiation degree and lymph node invasion. Furthermore, this research highlights the positive significant association between PMS2 loss and CDX2 loss, suggesting that CDX2 may be involved in molecular pathways that lead to loss of the MMR proteins. Despite the many studies performed, there are conflicting opinions related to the prognostic significance and the role of these molecular biomarkers in tumor progression. Future research is needed, in large representative series, to identify the most powerful prognostic panel of biomarkers in colon cancer in order to select high-risk patients and to find the most appropriate treatment algorithms.

\section{Conflict of interests}

The authors declare that they have no conflict of interests.

\section{References}

[1] Siegel R, Naishadham D, Jemal A. Cancer statistics, 2012. CA Cancer J Clin, 2012, 62(1):10-29. https://doi.org/10.3322/ caac.20138 PMID: 22237781

[2] Hagan S, Orr MCM, Doyle B. Targeted therapies in colorectal cancer - an integrative view by PPPM. EPMA J, 2013, 4(1):3. https://doi.org/10.1186/1878-5085-4-3 PMID: 23356214 PMCID: PMC3584939

[3] Ryan E, Sheahan K, Creavin B, Mohan HM, Winter DC. The current value of determining the mismatch repair status of colorectal cancer: a rationale for routine testing. Crit Rev Oncol Hematol, 2017, 116:38-57. https://doi.org/10.1016/j. critrevonc.2017.05.006 PMID: 28693799 
[4] Gatalica Z, Vranic S, Xiu J, Swensen J, Reddy S. High microsatellite instability (MSI-H) colorectal carcinoma: a brief review of predictive biomarkers in the era of personalized medicine. Fam Cancer, 2016, 15(3):405-412. https://doi.org/10.1007/ s10689-016-9884-6 PMID: 26875156 PMCID: PMC4901118

[5] Bray F, Ferlay J, Soerjomataram I, Siegel RL, Torre LA, Jemal A. Global cancer statistics 2018: GLOBOCAN estimates of incidence and mortality worldwide for 36 cancers in 185 countries. CA Cancer J Clin, 2018, 68(6):394-424. https://doi.org/10.3322/ caac.21492. Erratum in: CA Cancer J Clin, 2020, 70(4):313. PMID: 30207593

[6] Copija A, Waniczek D, Witkoś A, Walkiewicz K, NowakowskaZajdel E. Clinical significance and prognostic relevance of microsatellite instability in sporadic colorectal cancer patients. Int J Mol Sci, 2017, 18(1):107. https://doi.org/10.3390/ijms 18010107 PMID: 28067827 PMCID: PMC5297741

[7] Cunningham D, Atkin W, Lenz HJ, Lynch HT, Minsky B, Nordlinger B, Starling N. Colorectal cancer. Lancet, 2010 375(9719):1030-1047. https://doi.org/10.1016/S0140-6736(10) 60353-4 PMID: 20304247

[8] Ogino S, Nosho K, Kirkner GJ, Kawasaki T, Meyerhardt JA, Loda M, Giovannucci EL, Fuchs CS. CpG island methylator phenotype, microsatellite instability, $B R A F$ mutation and clinical outcome in colon cancer. Gut, 2009, 58(1):90-96. https://doi. org/10.1136/gut.2008.155473 PMID: 18832519 PMCID: PMC 2679586

[9] Shi C, Washington K. Molecular testing in colorectal cancer: diagnosis of Lynch syndrome and personalized cancer medicine. Am J Clin Pathol, 2012, 137(6):847-859. https://doi.org/10. 1309/AJCPI83DINULUJNI PMID: 22586043

[10] Parc Y, Gueroult S, Mourra N, Serfaty L, Fléjou JF, Tiret E, Parc R. Prognostic significance of microsatellite instability determined by immunohistochemical staining of $\mathrm{MSH} 2$ and MLH1 in sporadic T3N0M0 colon cancer. Gut, 2004, 53(3): 371-375. https://doi.org/10.1136/gut.2003.019190 PMID: 14960518 PMCID: PMC1773950

[11] Wheeler JM, Bodmer WF, Mortensen NJ. DNA mismatch repair genes and colorectal cancer. Gut, 2000, 47(1):148153. https://doi.org/10.1136/gut.47.1.148 PMID: 10861278 PMCID: PMC1727951

[12] Gologan A, Sepulveda AR. Microsatellite instability and DNA mismatch repair deficiency testing in hereditary and sporadic gastrointestinal cancers. Clin Lab Med, 2005, 25(1):179-196. https://doi.org/10.1016/j.cll.2004.12.001 PMID: 15749237

[13] Koncina E, Haan S, Rauh S, Letellier E. Prognostic and predictive molecular biomarkers for colorectal cancer: updates and challenges. Cancers (Basel), 2020, 12(2):319. https://doi. org/10.3390/cancers12020319 PMID: 32019056 PMCID: PMC7072488

[14] Bruegl AS, Ring KL, Daniels M, Fellman BM, Urbauer DL, Broaddus RR. Clinical challenges associated with universal screening for Lynch syndrome-associated endometrial cancer. Cancer Prev Res (Phila), 2017, 10(2):108-115. https://doi. org/10.1158/1940-6207.CAPR-16-0219 PMID: 27965287 PMCID: PMC5292079

[15] Haraldsdottir S, Hampel H, Tomsic J, Frankel WL, Pearlman R, de la Chapelle A, Pritchard CC. Colon and endometrial cancers with mismatch repair deficiency can arise from somatic, rather than germline, mutations. Gastroenterology, 2014, 147(6):13081316.e1. https://doi.org/10.1053/j.gastro.2014.08.041 PMID: 25194673 PMCID: PMC4294551

[16] Ichikawa Y, Lemon SJ, Wang S, Franklin B, Watson P, Knezetic JA, Bewtra C, Lynch HT. Microsatellite instability and expression of $\mathrm{MLH} 1$ and $\mathrm{MSH} 2$ in normal and malignant endometrial and ovarian epithelium in hereditary nonpolyposis colorectal cancer family members. Cancer Genet Cytogenet, 1999, 112(1):2-8. https://doi.org/10.1016/s0165-4608(98)00 252-0 PMID: 10432927

[17] McMeekin DS, Tritchler DL, Cohn DE, Mutch DG, Lankes HA, Geller MA, Powell MA, Backes FJ, Landrum LM, Zaino R, Broaddus RD, Ramirez N, Gao F, Ali S, Darcy KM, Pearl ML, DiSilvestro PA, Lele SB, Goodfellow PJ. Clinicopathologic significance of mismatch repair defects in endometrial cancer: an NRG Oncology/Gynecologic Oncology Group Study. J Clin Oncol, 2016, 34(25):3062-3068. https://doi.org/10.1200/JCO. 2016.67.8722 PMID: 27325856 PMCID: PMC5012715

[18] Segev Y, Pal T, Rosen B, McLaughlin JR, Sellers TA, Risch HA Zhang S, Sun P, Narod SA, Schildkraut J. Risk factors for ovarian cancers with and without microsatellite instability. Int $\mathrm{J}$ Gynecol Cancer, 2014, 24(4):664-669. https://doi.org/10.10 97/IGC.0000000000000134 PMID: 24755492

[19] Karpińska-Kaczmarczyk K, Lewandowska M, Ławniczak M, Białek A, Urasińska $E$. Expression of mismatch repair proteins in early and advanced gastric cancer in Poland. Med Sci Monit, 2016, 22:2886-2892. https://doi.org/10.12659/msm.897150 PMID: 27527654 PMCID: PMC4996049

[20] Mao L, Lee DJ, Tockman MS, Erozan YS, Askin F, Sidransky D. Microsatellite alterations as clonal markers for the detection of human cancer. Proc Natl Acad Sci U S A, 1994, 91(21): 9871-9875. https://doi.org/10.1073/pnas.91.21.9871 PMID: 7937908 PMCID: PMC44919

[21] Wadhwa N, Mathew BB, Jatawa SK, Tiwari A. Genetic instability in urinary bladder cancer: an evolving hallmark. J Postgrad Med, 2013, 59(4):284-288. https://doi.org/10.4103/ 0022-3859.123156 PMID: 24346386

[22] Shen C, Wang X, Tian L, Che G. Microsatellite alteration in multiple primary lung cancer. J Thorac Dis, 2014, 6(10):14991505. https://doi.org/10.3978/j.issn.2072-1439.2014.09.14 PMID: 25364529 PMCID: PMC4215141

[23] Kubecek O, Trojanova P, Molnarova V, Kopecky J. Microsatellite instability as a predictive factor for immunotherapy in malignant melanoma. Med Hypotheses, 2016, 93:74-76. https://doi.org/10.1016/j.mehy.2016.05.023 PMID: 27372860

[24] Ismael NEHS, EI Sheikh SA, Talaat SM, Salem EM. Mismatch repair proteins and microsatellite instability in colorectal carcinoma (MLH1, MSH2, MSH6 and PMS2): histopathological and immunohistochemical study. Open Access Maced J Med Sci, 2017, 5(1):9-13. https://doi.org/10.3889/oamjms.2017.003 PMID: 28293308 PMCID: PMC5320899

[25] Kawakami H, Zaanan A, Sinicrope FA. Microsatellite instability testing and its role in the management of colorectal cancer. Curr Treat Options Oncol, 2015, 16(7):30. https://doi.org/10. 1007/s11864-015-0348-2 PMID: 26031544 PMCID: PMC 4594190

[26] Richman S. Deficient mismatch repair: read all about it (review). Int J Oncol, 2015, 47(4):1189-1202. https://doi.org/10.3892/ ijo.2015.3119 PMID: 26315971 PMCID: PMC4583524

[27] Lindor NM, Burgart LJ, Leontovich O, Goldberg RM, Cunningham JM, Sargent DJ, Walsh-Vockley C, Petersen GM, Walsh MD, Leggett BA, Young JP, Barker MA, Jass JR, Hopper J, Gallinger S, Bapat B, Redston M, Thibodeau SN. Immunohistochemistry versus microsatellite instability testing in phenotyping colorectal tumors. J Clin Oncol, 2002, 20(4): 1043-1048. https://doi.org/10.1200/JCO.2002.20.4.1043 PMID: 11844828

[28] Bendardaf R, Elzagheid A, Lamlum H, Ristamäki R, Collan Y, Pyrhönen S. E-cadherin, CD44s and CD44v6 correlate with tumour differentiation in colorectal cancer. Oncol Rep, 2005 , 13(5):831-835. https://doi.org/10.3892/or.13.5.831 PMID: 15809746

[29] Kemler R. From cadherins to catenins: cytoplasmic protein interactions and regulation of cell adhesion. Trends Genet, 1993, 9(9):317-321. https://doi.org/10.1016/0168-9525(93) 90250-I PMID: 8236461

[30] Christou N, Perraud A, Blondy S, Jauberteau MO, Battu S, Mathonnet M. E-cadherin: a potential biomarker of colorectal cancer prognosis. Oncol Lett, 2017, 13(6):4571-4576. https:// doi.org/10.3892/ol.2017.6063 PMID: 28588719 PMCID: PMC5452924

[31] Jurčić $P$, Radulović $P$, Balja MP, Milošević $M$, Krušlin $B$. E-cadherin and NEDD9 expression in primary colorectal cancer, metastatic lymph nodes and liver metastases. Oncol Lett, 2019, 17(3):2881-2889. https://doi.org/10.3892/ol.2019. 9917 PMID: 30854064 PMCID: PMC6365943

[32] Tian X, Liu Z, Niu B, Zhang J, Tan TK, Lee SR, Zhao Y, Harris DC, Zheng G. E-cadherin/ $\beta$-catenin complex and the epithelial barrier. J Biomed Biotechnol, 2011, 2011:567305. https://doi.org/10.1155/2011/567305 PMID: 22007144 PMCID: PMC3191826

[33] Valenta T, Hausmann G, Basler K. The many faces and functions of $\beta$-catenin. EMBO J, 2012, 31(12):2714-2736. https://doi.org/10.1038/emboj.2012.150 PMID: 22617422 PMCID: PMC3380220

[34] Yang CM, Ji S, Li Y, Fu LY, Jiang T, Meng FD. $\beta$-Catenin promotes cell proliferation, migration, and invasion but induces apoptosis in renal cell carcinoma. Onco Targets Ther, 2017, 
10:711-724. https://doi.org/10.2147/OTT.S117933 PMID: 28260916 PMCID: PMC5328321

[35] Saad RS, Ghorab Z, Khalifa MA, Xu M. CDX2 as a marker for intestinal differentiation: its utility and limitations. World $J$ Gastrointest Surg, 2011, 3(11):159-166. https://doi.org/10. 4240/wjgs.v3.i11.159 PMID: 22180832 PMCID: PMC3240675

[36] Kaimaktchiev V, Terracciano L, Tornillo L, Spichtin H, Stoios D, Bundi M, Korcheva V, Mirlacher M, Loda M, Sauter G, Corless CL. The homeobox intestinal differentiation factor CDX2 is selectively expressed in gastrointestinal adenocarcinomas. Mod Pathol, 2004, 17(11):1392-1399. https://doi.org/10.1038/ modpathol.3800205 PMID: 15205684

[37] Werling RW, Yaziji H, Bacchi CE, Gown AM. CDX2, a highly sensitive and specific marker of adenocarcinomas of intestinal origin: an immunohistochemical survey of 476 primary and metastatic carcinomas. Am J Surg Pathol, 2003, 27(3):303310. https://doi.org/10.1097/00000478-200303000-00003 PMID: 12604886

[38] Munteanu O, Voicu D, Voiculescu DI, Negreanu L, Georgescu TA, Sajin M, Berceanu C, Mehedinţu C, Brătilă E, Istrate-Ofiţeru AM, Cîrstoiu MM. Colon cancer in pregnancy: a diagnostic and therapeutic challenge. Rom J Morphol Embryol, 2019, 60(1): 307-317. PMID: 31263861

[39] Sayar I, Akbas EM, Isik A, Gokce A, Peker K, Demirtas L, Gürbüzel M. Relationship among mismatch repair deficiency, CDX2 loss, p53 and E-cadherin in colon carcinoma and suitability of using a double panel of mismatch repair proteins by immunohistochemistry. Pol J Pathol, 2015, 66(3):246-253. https://doi.org/10.5114/pjp.2015.54958 PMID: 26619103

[40] Bosman FT, Carneiro F, Hruban RH, Theise ND (eds). World Health Organization (WHO) Classification of tumours of the digestive system. $4^{\text {th }}$ edition, International Agency of Research on Cancer (IARC) Press, Lyon, France, 2010, 131181.

[41] Karahan B, Argon A, Yıldırım M, Vardar E. Relationship between MLH-1, MSH-2, PMS-2, MSH-6 expression and clinicopathological features in colorectal cancer. Int $\mathrm{J}$ Clin Exp Pathol, 2015, 8(4):4044-4053. PMID: 26097592 PMCID: PMC4466979

[42] Weiser MR. AJCC $8^{\text {th }}$ edition: colorectal cancer. Ann Surg Oncol, 2018, 25(6):1454-1455. https://doi.org/10.1245/s10 434-018-6462-1 PMID: 29616422

[43] Sinicrope FA, Sargent DJ. Molecular pathways: microsatellite instability in colorectal cancer: prognostic, predictive, and therapeutic implications. Clin Cancer Res, 2012, 18(6): 15061512. https://doi.org/10.1158/1078-0432.CCR-11-1469 PMID: 22302899 PMCID: PMC3306518

[44] Florescu-Tenea RM, Kamal AM, Mitruţ P, Mitruţ R, llie DS, Nicolaescu AC, Marinescu SA. Colon cancer: clinical, macroscopic and microscopic aspects. Rom J Morphol Embryol, 2018, 59(4):1179-1188. PMID: 30845299

[45] Hall G, Clarkson A, Shi A, Langford E, Leung H, Eckstein RP, Gill AJ. Immunohistochemistry for PMS2 and MSH6 alone can replace a four antibody panel for mismatch repair deficiency screening in colorectal adenocarcinoma. Pathology, 2010, 42(5):409-413. https://doi.org/10.3109/00313025.2010.4938 71 PMID: 20632815

[46] Chen W, Swanson BJ, Frankel WL. Molecular genetics of microsatellite-unstable colorectal cancer for pathologists. Diagn Pathol, 2017, 12(1):24. https://doi.org/10.1186/s13000-0170613-8 PMID: 28259170 PMCID: PMC5336657

[47] Shia J. Immunohistochemistry versus microsatellite instability testing for screening colorectal cancer patients at risk for hereditary nonpolyposis colorectal cancer syndrome. Part I. The utility of immunohistochemistry. J Mol Diagn, 2008 , 10(4):293-300. https://doi.org/10.2353/jmoldx.2008.080031 PMID: 18556767 PMCID: PMC2438196

[48] Wang SM, Jiang B, Deng Y, Huang SL, Fang MZ, Wang Y. Clinical significance of $M L H 1 / M S H 2$ for stage II/III sporadic colorectal cancer. World J Gastrointest Oncol, 2019, 11(11): 1065-1080. https://doi.org/10.4251/wjgo.v11.i11.1065 PMID: 31798786 PMCID: PMC6883179

[49] Kang S, Na Y, Joung SY, Lee SI, Oh SC, Min BW. The significance of microsatellite instability in colorectal cancer after controlling for clinicopathological factors. Medicine (Baltimore), 2018, 97(9):e0019. https://doi.org/10.1097/MD. 0000000000010019 PMID: 29489646 PMCID: PMC5851768
[50] Yoon YS, Yu CS, Kim TW, Kim JH, Jang SJ, Cho DH, Roh SA, Kim JC. Mismatch repair status in sporadic colorectal cancer: immunohistochemistry and microsatellite instability analyses. J Gastroenterol Hepatol, 2011, 26(12):1733-1739. https:// doi.org/10.1111/j.1440-1746.2011.06784.x PMID: 21615788

[51] Ward R, Meagher A, Tomlinson I, O'Connor T, Norrie M, Wu R, Hawkins N. Microsatellite instability and the clinicopathological features of sporadic colorectal cancer. Gut, 2001, 48(6):821829. https://doi.org/10.1136/gut.48.6.821 PMID: 11358903 PMCID: PMC1728324

[52] Jung SH, Kim SH, Kim JH. Prognostic impact of microsatellite instability in colorectal cancer presenting with mucinous, signet-ring, and poorly differentiated cells. Ann Coloproctol, 2016, 32(2):58-65. https://doi.org/10.3393/ac.2016.32.2.58 PMID: 27218096 PMCID: PMC4865466

[53] Hashmi AA, Ali R, Hussain ZF, Faridi N, Khan EY, Bakar SMA Edhi MM, Khan M. Mismatch repair deficiency screening in colorectal carcinoma by a four-antibody immunohistochemical panel in Pakistani population and its correlation with histopathological parameters. World J Surg Oncol, 2017, 15(1):116. https://doi.org/10.1186/s12957-017-1158-8 PMID: 28651545 PMCID: PMC5485685

[54] Guastadisegni C, Colafranceschi M, Ottini L, Dogliotti E. Microsatellite instability as a marker of prognosis and response to therapy: a meta-analysis of colorectal cancer survival data. Eur J Cancer, 2010, 46(15):2788-2798. https://doi.org/10. 1016/j.ejca.2010.05.009 PMID: 20627535

[55] Popat S, Hubner R, Houlston RS. Systematic review of microsatellite instability and colorectal cancer prognosis. J Clin Oncol, 2005, 23(3):609-618. https://doi.org/10.1200/JCO. 2005.01.086 PMID: 15659508

[56] Bruun J, Kolberg M, Nesland JM, Svindland A, Nesbakken A, Lothe RA. Prognostic significance of $\beta$-catenin, E-cadherin, and SOX9 in colorectal cancer: results from a large populationrepresentative series. Front Oncol, 2014, 4:118. https://doi. org/10.3389/fonc.2014.00118 PMID: 24904831 PMCID: PMC 4033250

[57] Lugli A, Zlobec I, Minoo P, Baker K, Tornillo L, Terracciano L, Jass JR. Prognostic significance of the wnt signalling pathway molecules APC, beta-catenin and E-cadherin in colorectal cancer: a tissue microarray-based analysis. Histopathology, 2007, 50(4):453-464. https://doi.org/10.1111/j.1365-2559.2007. 02620.x PMID: 17448021

[58] Iwamoto M, Ahnen DJ, Franklin WA, Maltzman TH. Expression of beta-catenin and full-length APC protein in normal and neoplastic colonic tissues. Carcinogenesis, 2000, 21(11): 1935-1940. https://doi.org/10.1093/carcin/21.11.1935 PMID: 11062151

[59] Chung GG, Provost E, Kielhorn EP, Charette LA, Smith BL, Rimm DL. Tissue microarray analysis of beta-catenin in colorectal cancer shows nuclear phospho-beta-catenin is associated with a better prognosis. Clin Cancer Res, 2001, 7(12):4013-4020. PMID: 11751495

[60] Morin PJ. $\beta$-Catenin signaling and cancer. Bioessays, 1999, 21(12):1021-1030. https://doi.org/10.1002/(SICI)1521-1878 (199912)22:1<1021::AID-BIES6>3.0.CO;2-P PMID: 10580987

[61] Tsanou E, Peschos D, Batistatou A, Charalabopoulos A, Charalabopoulos K. The E-cadherin adhesion molecule and colorectal cancer. A global literature approach. Anticancer Res, 2008, 28(6A):3815-3826. PMID: 19189669

[62] Elzagheid A, Algars A, Bendardaf R, Lamlum H, Ristamaki R, Collan Y, Syrjanen K, Pyrhonen S. E-cadherin expression pattern in primary colorectal carcinomas and their metastases reflects disease outcome. World J Gastroenterol, 2006, 12(27):4304-4309. https://doi.org/10.3748/wjg.v12.i27.4304 PMID: 16865770 PMCID: PMC4087739

[63] Hajra KM, Fearon ER. Cadherin and catenin alterations in human cancer. Genes Chromosomes Cancer, 2002, 34(3): 255-268. https://doi.org/10.1002/gcc.10083 PMID: 12007186

[64] Nazemalhosseini Mojarad E, Kashfi SMH, Mirtalebi H, Almasi S, Chaleshi V, Kishani Farahani R, Tarban P, Molaei M, Zali MR, Kuppen PJK. Prognostic significance of nuclear $\beta$-catenin expression in patients with colorectal cancer from Iran. Iran Red Crescent Med J, 2015, 17(7):e22324. https://doi.org/10. 5812/ircmj.22324v2 PMID: 26421170 PMCID: PMC4584109

[65] Al Khatib AM, Mărgăritescu C, Taisescu O, Andreiana BC, Florescu MM, Ciurea RN. Immunoexpression of E-cadherin, Snail and Twist in colonic adenocarcinomas. Rom J Morphol Embryol, 2019, 60(2):531-536. PMID: 31658326 
[66] Takayama T, Shiozaki H, Shibamoto S, Oka H, Kimura Y, Tamura S, Inoue M, Monden T, Ito F, Monden M. Beta-catenin expression in human cancers. Am J Pathol, 1996, 148(1):39 46. PMID: 8546224 PMCID: PMC1861624

[67] Wong SCC, Lo ESF, Chan AKC, Lee KC, Hsiao WL. Nuclear beta catenin as a potential prognostic and diagnostic marker in patients with colorectal cancer from Hong Kong. Mol Pathol, 2003, 56(6):347-352. https://doi.org/10.1136/mp.56.6.347 PMID: 14645698 PMCID: PMC1187354

[68] Kanczuga-Koda L, Wincewicz A, Fudala A, Abrycki T, Famulski W, Baltaziak M, Sulkowski S, Koda M. E-cadherin and $\beta$-catenin adhesion proteins correlate positively with connexins in colorectal cancer. Oncol Lett, 2014, 7(6):18631870. https://doi.org/10.3892/ol.2014.1970 PMID: 24932249 PMCID: PMC4049722

[69] Ahmad A, Hahn-Stromberg V. Expression of $\beta$-catenin and E-cadherin, their clinical significance and association with complexity index of colon carcinoma. Adv Genet Eng, 2016, 5(3):1000156. https://doi.org/10.4172/2169-0111.1000156

[70] Walther A, Johnstone E, Swanton C, Midgley R, Tomlinson I, Kerr D. Genetic prognostic and predictive markers in colorectal cancer. Nat Rev Cancer, 2009, 9(7):489-499. https://doi.org/ 10.1038/nrc2645. Erratum in: Nat Rev Cancer, 2011, 11(4): 309. PMID: 19536109

[71] Iseki Y, Shibutani M, Maeda K, Nagahara H, Ikeya T, Hirakawa K. Significance of E-cadherin and CD44 expression in patients with unresectable metastatic colorectal cancer. Oncol Lett, 2017, 14(1):1025-1034. https://doi.org/10.3892 ol.2017.6269 PMID: 28693269 PMCID: PMC5494689

[72] Gao ZH, Lu C, Wang MX, Han Y, Guo LJ. Differential $\beta$ catenin expression levels are associated with morphologica features and prognosis of colorectal cancer. Oncol Lett, 2014, 8(5):2069-2076. https://doi.org/10.3892/ol.2014.2433 PMID: 25295092 PMCID: PMC4186582

[73] Li Z, Yin S, Zhang L, Liu W, Chen B. Prognostic value of reduced E-cadherin expression in breast cancer: a metaanalysis. Oncotarget, 2017, 8(10):16445-16455. https://doi org/10.18632/oncotarget.14860 PMID: 28147315 PMCID: PMC5369975

[74] Chen J, Zhao J, Ma R, Lin H, Liang X, Cai X. Prognostic significance of E-cadherin expression in hepatocellular carcinoma: a meta-analysis. PLoS One, 2014, 9(8):e103952. https:// doi.org/10.1371/journal.pone.0103952 PMID: 25093414 PMCID: PMC4122395

[75] Garcia del Muro X, Torregrosa A, Muñoz J, Castellsagu X, Condom E, Vigués F, Arance A, Fabra A, Germà JR. Prognostic value of the expression of E-cadherin and betacatenin in bladder cancer. Eur J Cancer, 2000, 36(3):357362. https://doi.org/s0959-8049(99)00262-2 PMID: 10708937

[76] van Oort IM, Tomita K, van Bokhoven A, Bussemakers MJG Kiemeney LA, Karthaus HFM, Witjes JA, Schalken JA. The prognostic value of E-cadherin and the cadherin-associated molecules alpha-, beta-, gamma-catenin and p120ctn in prostate cancer specific survival: a long-term follow-up study. Prostate, 2007, 67(13):1432-1438. https://doi.org/10.1002/ pros.20626 PMID: 17639504

[77] Zacapala-Gómez AE, Navarro-Tito N, Alarcón-Romero LDC Ortuño-Pineda C, Illades-Aguiar B, Castañeda-Saucedo E, Ortiz-Ortiz J, Garibay-Cerdenares OL, Jiménez-López MA, Mendoza-Catalán MA. Ezrin and E-cadherin expression profile in cervical cytology: a prognostic marker for tumor progression in cervical cancer. BMC Cancer, 2018, 18(1):349. https://doi. org/10.1186/s12885-018-4243-7 PMID: 29587669 PMCID: PMC5872531
[78] Tomasello G, Barni S, Turati L, Ghidini M, Pezzica E, Passalacqua R, Petrelli F. Association of CDX2 expression with survival in early colorectal cancer: a systematic review and meta-analysis. Clin Colorectal Cancer, 2018, 17(2):97-103. https://doi.org/10.1016/j.clcc.2018.02.001 PMID: 29519711

[79] Bae JM, Lee TH, Cho NY, Kim TY, Kang GH. Loss of CDX2 expression is associated with poor prognosis in colorectal cancer patients. World J Gastroenterol, 2015, 21(5):14571467. https://doi.org/10.3748/wjg.v21.i5.1457 PMID: 25663765 PMCID: PMC4316088

[80] Graule J, Uth K, Fischer E, Centeno I, Galván JA, Eichmann M, Rau TT, Langer R, Dawson H, Nitsche U, Traeger P, Berger MD, Schnüriger $B$, Hädrich $M$, Studer $P$, Inderbitzin $D$, Lugli $A$, Tschan MP, Zlobec I. CDX2 in colorectal cancer is an independent prognostic factor and regulated by promoter methylation and histone deacetylation in tumors of the serrated pathway. Clin Epigenetics, 2018, 10(1):120. https://doi.org/ 10.1186/s13148-018-0548-2 PMID: 30257705 PMCID: PMC 6158822

[81] Baba Y, Nosho K, Shima K, Freed E, Irahara N, Philips J Meyerhardt JA, Hornick JL, Shivdasani RA, Fuchs CS, Ogino S. Relationship of CDX2 loss with molecular features and prognosis in colorectal cancer. Clin Cancer Res, 2009, 15(14):4665-4673. https://doi.org/10.1158/1078-0432.CCR09-0401 PMID: 19584150 PMCID: PMC2777758

[82] Altintas S, Bayrak M, Altintas Y. Prognostic value of CDX2 and villin expression in advanced stage colorectal carcinoma. J Coll Physicians Surg Pak, 2019, 29(11):1057-1061. https:// doi.org/10.29271/jcpsp.2019.11.1057 PMID: 31659962

[83] Olsen J, Espersen MLM, Jess P, Kirkeby LT, Troelsen JT. The clinical perspectives of CDX2 expression in colorectal cancer: a qualitative systematic review. Surg Oncol, 2014, 23(3):167-176. https://doi.org/10.1016/j.suronc.2014.07.003 PMID: 25126956

[84] Okoń K, Zazula M, Rudzki Z, Papla B, Osuch C, Stachura J. CDX-2 expression is reduced in colorectal carcinomas with solid growth pattern and proximal location, but is largely independent of MSI status. Pol J Pathol, 2004, 55(3):9-14. PMID: 15619975

[85] Cecchini MJ, Walsh JC, Parfitt J, Chakrabarti S, Correa RJ, MacKenzie MJ, Driman DK. CDX2 and Muc2 immunohistochemistry as prognostic markers in stage II colon cancer. Hum Pathol, 2019, 90:70-79. https://doi.org/10.1016/j.hum path.2019.05.005 PMID: 31121192

[86] Hong KD, Lee D, Lee Y, Lee SI, Moon HY. Reduced CDX2 expression predicts poor overall survival in patients with colorectal cancer. Am Surg, 2013, 79(4):353-360. PMID: 23574843

[87] Slik K, Turkki R, Carpén O, Kurki S, Korkeila E, Sundström J, Pellinen T. CDX2 loss with microsatellite stable phenotype predicts poor clinical outcome in stage II colorectal carcinoma. Am J Surg Pathol, 2019, 43(11):1473-1482. https://doi.org/ 10.1097/PAS.0000000000001356 PMID: 31490234

[88] Ryan ÉJ, Creavin B, Khaw YL, Kelly ME, Mohan HM, Geraghty R, Ryan EJ, Kennelly R, Hanly A, Martin ST, Fennelly D, McDermott R, Gibbons D, O'Connell PR, Sheahan K, Winter DC. Effects of CDX2 on prognosis and chemotherapy responsiveness in mismatch repair-deficient colorectal cancer. BJS Open, 2018, 2(6):456-463. https:// doi.org/10.1002/bjs5.91 PMID: 30511046 PMCID: PMC 6253792

\section{Corresponding author}

Adina Bianca Boşca, Associate Professor, MD, PhD, Discipline of Histology, Department of Morphological Sciences, Iuliu Haţieganu University of Medicine and Pharmacy, 4-6 Louis Pasteur Street, 400349 Cluj-Napoca, Romania; Phone +40740-248 923, e-mail: biancabosca@yahoo.com 